\title{
Phase Field Simulation of Charged Interface Formation during Ferroelectric Switching
}

Jason Britson ${ }^{\mathrm{a}}$, Peng Gao ${ }^{\mathrm{b}}$, Xiaoqing Pan ${ }^{\mathrm{b}}$, and Long-Qing Chen ${ }^{\mathrm{a}}{ }^{\dagger}$

${ }^{a}$ Department of Materials Science and Engineering, The Pennsylvania State University, University Park, PA 16802

${ }^{\mathrm{b}}$ Department of Materials Science and Engineering, University of Michigan, Ann Arbor, MI 48109, USA

$\uparrow$ - Author to whom correspondence should be addressed

E-mail: lqc3@psu.edu

\begin{abstract}
Ferroelectric switching in thin films is a local process strongly influenced by the presence and spatial distribution of defects such as dislocations, grain boundaries and preexisting domains. Preexisting ferroelastic domains have been shown to inhibit $180^{\circ}$ switching in (001)-oriented epitaxial $\mathrm{Pb}\left(\mathrm{Zr}_{0.2}, \mathrm{Ti}_{0.8}\right) \mathrm{O}_{3}(\mathrm{PZT})$ films, but ferroelectric switching pathways around these domains remain unclear. Here, phase field modeling with supporting high resolution transmission electron microscopy is used to investigate ferroelectric switching in PZT thin films around such a ferroelastic domain. We show $180^{\circ}$ domain wall motion is arrested at moderate applied biases by the ferroelastic domain through formation of charged $90^{\circ}$ domain walls during switching. This leads to an increased applied bias required for complete switching through the thin film. Charged $90^{\circ}$ ferroelastic domain walls are found to be partially stabilized by local rotation of the polarization direction and significant broadening of the head-to-head wall to distribute the bound charge, leading to domain walls $5-6 \mathrm{~nm}$ in thickness compared to $0.5-2 \mathrm{~nm}$ for typical $90^{\circ}$ domain walls. Ferroelectric switching continues only at higher applied electric fields. This study provides a more complete picture of the ferroelectric switching pathway around ferroelastic domains than has been previously available and may explain the experimentally observed lower stability of written domain structures around preexisting ferroelastic domain structures in epitaxial ferroelectric thin films.
\end{abstract}

Keywords: Ferroelectric; Domain Boundaries; Phase Field Modeling; Thin Films; Domain Switching 


\section{Introduction}

Complete switching of a polarization state is critical to a number of applications for ferroelectric thin films. Typical epitaxial thin films contain myriad domains each with an individual polarization state that are separated by domain walls with intermediate polarization. Reorienting the polarization in a ferroelectric crystal generally requires significant motion of these domain wall and a reduced switchable component of the polarization has been observed in systems with only limited domain wall mobility.[1] In perovskite ferroelectric thin films $180^{\circ}$ domain wall motion has been widely reported to be inhibited by the existence of various defects in a film such as dislocations, [2-4] free charge carriers,[5-7] grain boundaries, [8] or preexisting ferroelastic domains,[9] which are able to locally pin domain walls through elastic and electrostatic interactions or screening of applied electric fields. With recent advancements in the quality of epitaxial thin films, however, concentrations of many of these defects can be reduced or eliminated in epitaxial $\mathrm{Pb}\left(\mathrm{Zr}_{0.2}, \mathrm{Ti}_{0.8}\right) \mathrm{O}_{3}(\mathrm{PZT})$ thin films. Above a critical thickness of about $100 \mathrm{~nm}$, however, ferroelastic domains with a polarization rotated by $90^{\circ}$ form dense domain structures to reduce elastic coherency strain in the film.[10,11] Due to the strong elastic driving force for formation of ferroelastic domains, this type of defect is difficult to avoid during film fabrication. As a result, understanding the ferroelectric switching behavior of ferroelastic domains in thin films is essential to the development of ferroelectrics for microelectronic applications.

Several studies have shown ferroelastic domains play critical roles in polarization switching in epitaxial PZT thin films. [1,10,12-17] Preexisting ferroelastic domains are often immobile in constrained thin films $[3,9,18]$ and inhibit complete ferroelectric switching when a film is poled. $[10,12,13]$ Therefore, substantial increases in the remnant polarization and 
dielectric response can be achieved by increasing the mobility of these domains $[1,15,16]$ or removing them entirely. [19] Internal ferroelastic domains have also been reported to degrade performance of ferroelectric devices by decreasing the stability of switched domains and rapid back-switching of written domains to the original domain configuration has been observed to begin near ferroelastic domain walls in PZT thin films. [17]

The mechanism by which preexisting ferroelastic domains inhibit $180^{\circ}$ switching and reduce the stability of written domain structures in PZT thin films remains unclear, however. Several studies have suggested that trapping of point defect dipoles around ferroelastic domains creates a built-in electric field that reduces the coercive field to back switching.[7,19,20] Quenching a random dipole structure in $\mathrm{BiFeO}_{3}$ thin films, for instance, has been shown to reduce back switching of ferroelectric domain structures.[7,16] Defect-induced dipoles, however, may not exist in high densities in annealed thin films with low concentrations of oxygen vacancies. Other studies have indicated that back switching around $90^{\circ}$ domain structures in tetragonal PZT is the result of depolarization fields created by incomplete switching of preexisting ferroelastic domains.[17,21] These depolarization fields were thought to be created by the formation of charged $90^{\circ}$ domain walls between switched domains and preexisting ferroelastic domain walls. No charged domain walls, however, were directly observed in these studies since only surface sensitive techniques were used.

To overcome this limitation Gao, et al [12] used high resolution transmission electron microscopy to observe in situ ferroelectric switching around an embedded ferroelastic domain. In contrast to previous results, charged $90^{\circ}$ domain walls formed during the switching process were found to remain stable once the applied electric field was removed. Tan and Shang [22] showed similar charged $90^{\circ}$ domain walls between ferroelastic domains were created by the 
intersection of ferroelastic domain during switching of PMN-PT bulk crystals. These systems, however, appeared to be influenced by local dislocations noted in both studies. Tan and Shang, in particular, directly observed the creation of partial dislocations at the charged interface. Once stabilized by dislocations, the charged interfaces did not immediately revert to the original domain structure in the thin film. Ferroelectric switching behaviors in these films are clearly dependent on creation of charged $90^{\circ}$ interfaces, but switching near ferroelastic domains in thin films free of dislocations remains poorly understood.

In this manuscript we report on phase field modeling and high resolution transmission electron microscopy of switching around an embedded ferroelastic domain in a $\mathrm{Pb}\left(\mathrm{Zr}_{0.2}, \mathrm{Ti}_{0.8}\right) \mathrm{O}_{3}$ epitaxial thin film using an inhomogeneous electric field generated by a local probe. Formation of transient charged domain walls at preexisting ferroelastic domains is observed during ferroelectric switching around an immobile ferroelastic domain. These charged domain walls are found to persist over a range of applied biases even without local dislocations, inhibiting complete switching through the thickness of the film at small applied electrical biases. The relative stability of charged $90^{\circ}$ domain walls during switching is demonstrated to be the result of a reduction in local depolarization electric field at the domain wall associated with broadening of the charge distribution at the interface, which reduces the electrostatic energy and results in a partially stabilized domain wall. These results help explain back switching caused by incompletely switched $90^{\circ}$ domains [17] and have implications for the role high-energy, charged domain interfaces have in the switching dynamics of ferroelectric thin films. 


\section{Simulation Method}

Evolution of the ferroelectric domain structures during switching was modeled in an epitaxial, (001)-oriented $\mathrm{Pb}\left(\mathrm{Zr}_{0.2}, \mathrm{Ti}_{0.8}\right) \mathrm{O}_{3}$ thin film using the phase field approach.[23-25] Polar domains in the system were described using a continuous vector field representing the three principal components of polarization in the system. Evolution of this vector field with time, $t$, during ferroelectric switching was modeled by solving the time dependent Ginzburg-Landau equations $[24,26,27]$

$$
\frac{\partial P_{i}}{\partial t}=-L \frac{\delta F}{\delta P_{i}},
$$

which evolve the components of the polarization, $P_{\mathrm{i}}$, toward the minimum in the free energy of the thin film, $F$, with respect to the distribution of polarization in the film. In Equation $1 L$ is a kinetic coefficient related to the domain wall mobility of the system. Ferroelectric domain structures were modeled in a coherent thin film on a thick substrate that was allowed to relax for a short distance beneath the thin film. [27] Allowing the substrate to partially relax is necessary to accommodate the domain structure in the thin film. A short distance from the film interface, the substrate was assumed to reach a bulk, unperturbed state. The system was also assumed to be much larger in lateral extent than in thickness and as a result periodic boundary conditions were used in the two in-plane directions while specific boundary conditions were used in the outof-plane direction.

Total free energy in Equation 1 consists of four contributions, the bulk free energy, $f_{\text {bulk; }}$; the gradient energy, $f_{\text {gradient }}$; the electrostatic interaction energy, $f_{\text {electric }}$; and the elastic interaction energy, $f_{\text {elastic }}$, integrated over the volume of the film, $V$ :

$$
F=\int\left[f_{\text {bulk }}+f_{\text {gradient }}+f_{\text {electric }}+f_{\text {elastic }}\right] d V .
$$


The bulk free energy density was used to describe the local energy of the stress free crystal using the sixth order Landau polynomial

$$
f_{\text {bulk }}=\alpha_{i} P_{i}^{2}+\beta_{i j} P_{i}^{2} P_{j}^{2}+\gamma_{i j k} P_{i}^{2} P_{j}^{2} P_{k}^{2}
$$

with the phenomenological coefficients $\alpha_{\mathrm{i}}, \beta_{\mathrm{ij}}$, and $\gamma_{\mathrm{ijk}}$ measured by Haun, et al.[28] Repeated indices in Equation 3 indicate summation over the repeated indices. For simplicity the gradient energy was assumed to be isotropic, $[29,30]$ leading to the expression for gradient energy of

$$
f_{\text {gradient }}=G_{i j}\left(\partial_{j} P_{i}\right)^{2} .
$$

Interactions between bound charges in the system lead to the electrostatic energy density in the system $[31,32]$

$$
f_{\text {electric }}=-E_{i} P_{i}-\frac{1}{2} \varepsilon_{0} \kappa_{i j} E_{i} E_{j} .
$$

where $E_{\mathrm{i}}$ is the $i^{\text {th }}$ component of the total electric field from the applied electric field and the depolarizing electric field, $E_{i}^{b}$, created by bound charges around the polarization distribution. In Equation $5 \varepsilon_{0}$ is the permittivity of free space and $\kappa_{\mathrm{ij}}$ is the background dielectric constant of PZT, [33] which is assumed here to be isotropic and have a value of 100 . Values of the dielectric constant between 10 and 200 were also tested and found to yield qualitatively similar results, but a thorough discussion of the effects of the dielectric constant on the phase field simulation is beyond the scope of this article. Electric fields around bound charges are determined by first solving the Poisson equation, $\varepsilon_{0} \partial_{i}\left(\kappa_{i i} \partial_{i} \varphi\right)=\partial_{i} P_{i}$, for the electric potential, $\varphi$, and then using the relationship $E_{i}^{b}=-\partial_{i} \varphi$. For simplicity, fully compensating electrodes with negligible screening lengths were assumed so that boundary conditions for the Poisson equation fixed the potential at both surfaces of the film.

Elastic strain energy introduced by the spontaneous deformation associated with the ferroelectric transition was described using the expression $[27,34]$ 


$$
f_{\text {elastic }}=\frac{1}{2} C_{i j k l}\left(\varepsilon_{i j}-\varepsilon_{i j}^{0}\right)\left(\varepsilon_{k l}-\varepsilon_{k l}^{0}\right)
$$

where $\varepsilon_{i j}$ is the total local strain in the thin film and $\varepsilon_{i j}^{0}$ is the spontaneous non-elastic strain due to the ferroelectric transformation. The spontaneous strain is related to the electrostrictive effect in the ferroelectric and is defined as a function of the spontaneous polarization as [35] $\varepsilon_{i j}^{0}=$ $Q_{i j k l} P_{k} P_{l}$ where $Q_{i j k l}$ is the electrostrictive coefficient tensor.[28] Both total strain and spontaneous strain are defined with the cubic paraelectric phase as a reference. Strain fields created by spontaneous deformations in the system were found by solving the mechanical equilibrium equations $C_{i j k l} \partial_{j}\left(\varepsilon_{k l}-\varepsilon_{k l}^{0}\right)=0$ where $C_{i j k l}$ is the elastic stiffness tensor. [23,27] To model the coherent thin film the out of plane components of the stress were assumed to be zero at the top surface of the thin film while the mechanical displacement was assumed to be zero at the bottom of the substrate volume included in the simulation. Solution of the mechanical equilibrium problem in thin films is described in more depth in previous publications. [24,27]

The ferroelectric thin film was modeled on a discrete grid of size $256 \Delta \times 48 \Delta \times 128 \Delta$ with a regular spacing between grid points, $\Delta$, of $1.0 \mathrm{~nm}$. Film thickness was assumed to be 96 $\mathrm{nm}$ with a $14 \mathrm{~nm}$ thickness of substrate allowed to elastically relax. This substrate thickness has been shown to be sufficiently large to ensure the elastic constraints on the substrate imposed by the boundary conditions do not significantly alter the domain structure in the ferroelectric thin film.[27] An average compressive strain of $-0.7 \%$ was assumed to be applied by the substrate to represent partial relaxation of the compressive coherency strain imposed on a PZT thin film by a single crystal perovskite substrate, such as $\mathrm{SrTiO}_{3}$ or $\mathrm{DyScO}_{3}$, through misfit dislocation 
formation at the interface.[36] Strain fields around these dislocations, however, were not explicitly considered in the model for simplicity.

Distributions of the polarization components in the system were evolved by solving Equation 1 using the semi-implicit Fourier-Spectral method described in References [25,27,37] with the thin film boundary conditions described above. For the solution the gradient energy coefficient, $G_{\mathrm{ij}}$, in Equation 4 was assumed to be $9.0 \times 10^{-11} \mathrm{~N} \mathrm{~m}^{4} \mathrm{C}^{-2}$, which produced a $90^{\circ}$ domain wall width of $1-2 \mathrm{~nm}$ and was in reasonable agreement with experimental observations.[12,38,39] A time step of $\Delta t / t_{0}=0.01$ was used, where $t_{0}=\left(L\left|\alpha_{i}\right|_{T=25^{\circ} \mathrm{C}}\right)^{-1}$, that ensured the system remained stable, but was large enough to allow the domain structure to quickly evolve toward equilibrium. To simulate switching around an isolated ferroelastic domain in a film with the polarization primarily out of the plane of the film and a single ferroelastic domain with the polarization in the plane of the film was considered. This domain structure was evolved with no applied potential until equilibrium was reached. The stable domain configuration is shown schematically in Figure 1a.

\section{Results}

Dynamic switching behavior around an embedded ferroelastic domain was modeled with an applied electric field created by fixing the applied potential at the top surface of the thin film while the potential at the bottom surface was held at zero. Here, a Lorentz-like distribution of the applied potential, $\varphi_{a p p}$, with the form[40]

$$
\varphi_{a p p}=\varphi_{0} \frac{\gamma^{2}}{\gamma^{2}+r^{2}}
$$

was used to model switching under a small electrode tip (see Figure 1a). In Equation $7 r$ describes the distance between the center of the applied potential distribution and a point on the 
thin film surface and $\gamma$ describes the width of the applied potential distribution that was assumed to have a value of $10.0 \mathrm{~nm}$. Initial stages of switching were simulated through application of a potential at the top surface of the thin film that increased from $0 \mathrm{~V}$ to $3.0 \mathrm{~V}$ in increments of 37 $\mathrm{mV}$. This small increment was chosen to ensure the potential in the system changed smoothly.

Phase field simulations show ferroelectric switching occurs directly underneath the electrode where the electric field is strongest (Figure 1), not near the ferroelastic domain wall, which has been reported to behave as a heterogeneous nucleation site for local switching.[40] Initial growth of the switched region occurred through elongation of the needle shaped domain with little lateral expansion in order to minimize the bound charge around the new domain. Once the $P_{[00 \overline{1}]}$ domain reached the stationary ferroelastic domain, however, rapid forward growth was arrested by the formation of a positively charged head-to-head domain wall with the existing $P_{[100]}$ ferroelastic domain. This stationary ferroelastic domain acted as a persistent obstacle to continued forward switching through the film and the charged domain wall was observed to be stable over a small range of biases. Continued switching through the thickness of the thin film either by removal of the ferroelastic domain[15] or $180^{\circ}$ switching of the ferroelastic domain[12] was not observed here at moderate biases. Rather, at moderate biases ferroelectric switching in the thin film continued by lateral growth of the switched domain as the applied bias was increased, expanding the interfacial area of the charged domain wall interface.

Previous work has shown that saw-tooth domain structures with interlaced fingers of $P_{[001]}$ and $P_{[100]}$ domains form to reduce the charge at the interface. This structure has been reported to locally establish more nearly head-to-tail domain walls and distribute the total bound charge in the system.[9] Here, however, the head-to-head interface appears to remain straight, likely due to the high gradient energy that would be associated with forming a saw-tooth domain 
structure. The shape and orientation of the preexisting ferroelastic domain also does not change significantly during the switching process and the domain wall remains oriented primarily along the (101) plane. Restructuring of the domain wall is expected to be unlikely due to the large elastic strain energy associated with bending the domain wall away from the (101) plane that forms a mechanically compatible interface between the $P_{[100]}$ and $P_{[001]}$ domains. Lack of relaxations in the domain structure to accommodate local electric fields, however, contrasts with previous simulations of $\mathrm{BiFeO}_{3}$ thin films showing domain wall twisting in ferroelectric thin films occurred to reduce the energy associated with depolarization fields within the film.[41] To investigate changes to the domain wall structure around the head-to-head charged domain wall, the magnitude of the polarization and projection of the polarization onto the plane directly beneath the electrode were calculated and are shown in Figure 2. Figure 2a shows that prior to formation of the head-to-head domain wall both $90^{\circ}$ ferroelastic domain walls in the system are sharp and the transition between the $P_{[001]}$ and $P_{[\overline{1} 00]}$ domains occurs over $1-2 \mathrm{~nm}$ with the polarization perpendicular to the domain wall plane at the center of the wall. This transition over a small number of unit cells is consistent with experimental observations of similar domain walls in PZT thin films. $[38,39,42]$ Once the head-to-head domain wall forms, however, the structure of the $90^{\circ}$ ferroelastic domain wall in the simulation becomes more diffuse than the surrounding head-to-tail ferroelastic domain walls (Figure 2b). Expanded views of both the charged head-to-head and uncharged head-to-tail domain wall structures are shown in Figures $2 \mathrm{c}$ and $2 \mathrm{~d}$, respectively. The structure of the head-to-tail domain wall remains unchanged during switching at moderate applied electric fields. In contrast, the polarization direction in the head-to-head domain wall rotates gradually between $P_{[00 \overline{1}]}$ and $P_{[\overline{1} 00]}$ polarizations so that the polarization at the center of the domain wall is along the unit cell body 
and diagonal and is parallel to the domain wall plane. This transition is also seen to occur over a diffuse domain wall region.

Line traces across the domain wall of the polarization components normal and transverse to the domain wall plane show that the component of the polarization transverse to the domain wall remains nearly constant across the charged head-to-head $90^{\circ}$ domain wall while the normal component changes sign across the domain boundary (Figure 2e). Only a slight increase in the magnitude of the transverse polarization is also observed across the charged boundary so that the net magnitude of the polarization decreases less dramatically at the charged domain wall than the charge neutral domain walls, suggesting that the polarization rotated to along the body diagonal of the unit cell is a lower energy configuration than a suppressed or randomized polarization state. Similarly rotated polarization has been previously observed in constrained, tetragonal PZT thin films, $[43,44]$ clearly showing that the $P_{[101]}$ state is an energetically accessible polarization in some thin films. Transition between the domains on either side of the charged wall occurs over a distance of $5-6 \mathrm{~nm}$, which corresponds to approximately $10-12$ unit cells.[45] The polarization distribution observed at the charged $90^{\circ}$ domain wall is considerably different from predicted equilibrium domain structures where a constant normal polarization component is required to produce a charge neutral domain wall. Similarly diffuse transitions regions between domains that form charged domain walls have been reported in high resolution transmission electron microscopy (HRTEM) of charged domain walls either in as-grown thin films [38] or that form during ferroelastic switching. [9,12]

We validated the results of our simulations with high resolution transmission electron microscopy (HRTEM) of in-situ domain switching in PZT 20/80 thin films similar to the simulation system. Thin epitaxial film samples for microscopy were prepared on well lattice- 
matched $\mathrm{DyScO}_{3}$ substrates using a $90^{\circ}$ off-axis sputtering thin film growth method as described in our previous publications. [2,12,44] Prior to depositing a PZT thin film a $20 \mathrm{~nm}$ thickness of $\mathrm{SrRuO}_{3}$ was deposited as a buffer layer and bottom electrode for the sample. The $\mathrm{SrRuO}_{3}$ was grown at $560^{\circ} \mathrm{C}$ in an atmosphere at 200 mTorr consisting of $\mathrm{Ar}$ and $\mathrm{O}_{2}$ at a ratio of $3: 2$. This was followed by growth of a $200 \mathrm{~nm}$ thickness of PZT $20 / 80$ grown at $530^{\circ} \mathrm{C}$ with a $\mathrm{Ar}: \mathrm{O}_{2}$ ratio of 1:1. TEM samples were prepared from the films by cross sectioning the samples followed by mechanical polishing and Ar ion milling to electron transparency. The in-situ switching experiments were carried out in a JEOL 2010F microscope. High-resolution HAADF-STEM images were obtained from aberration corrected FEI Titan operating at $300 \mathrm{kV}$ with a semiconvergence angle of $17 \mathrm{mrad}$, collection angles of $[53 ; 270] \mathrm{mrad}$ and beam current of $0.07 \mathrm{nA}$. Electric fields were applied to study in-situ switching behavior of the ferroelectric domains using a custom tungsten-tip to act as a mobile electrode. [12]

The observed domain structure of this film was a mixture of out-of-plane and in-plane oriented ferroelastic domains similar to the simulation system (Figure 3a.) However, the out-ofplane polarization in some films studied was switched to down, rather than up. This was likely the result of built-in biases in the film due to electronic contacts and adsorbed surface ions. As a result, negative, rather than positive, biases were applied to induce switching in the thin film. This did not appear to qualitatively change switching behavior of domains in the thin films around embedded ferroelastic domains as evidenced by similar behavior between the simulations and thin films.

During in-situ switching of the PZT thin film near a $90^{\circ}$ ferroelastic domain a charged $90^{\circ}$ domain wall was observed as an intermediate switching step, as shown in Figures $3 \mathrm{a}$ and $3 \mathrm{~b}$. Despite the significant electrostatic charges associated with this domain structure, this structure 
was stable for several minutes once the applied bias was removed, indicating an additional stabilization mechanism at the domain wall. Mapping of the polarization structure in a high resolution image of the charged boundary using the observed lattice plane spacing, as discussed in previous publications, $[12,46]$ reveals that the charged domain boundary developed a wide transition region with intermediate polarization between the two ferroelectric domains in contrast to the narrow transition regions in charged-neutral $90^{\circ}$ domain boundaries. Figure $3 \mathrm{c}$ shows this situation for a head-to-head charged domain wall observed at a similar ferroelastic domain boundary in the thin film. The observed broad transition region indicates that competition between local electrostatic charge and Landau energy of the crystal may lead to local deviations of the polarization away from the lowest energy bulk configurations to reduce energy rather than large motions of the domain wall to locally stabilize the structure. Relative stability of this structure in both the in-situ observations and simulation results from the decrease in energy compared to nearby states achieved by disordering the polarization structure at the domain wall as discussed below.

\section{Discussion}

Stability of the charged domain wall to further ferroelectric switching was investigated by calculating the induced electric fields and associated electrostatic energy around the domain structure both before and after formation of the charged head-to-head domain wall. Prior to formation of the charged domain wall, large depolarization fields are present around the switched ferroelastic domain due to the bound charges around the $180^{\circ}$ domain wall, as shown in Figure 4. Figures $4 \mathrm{a}$ and $4 \mathrm{c}$ show that strong fields exist below the switched domain in the out-of-plane direction of the film and on either lateral side of the switched domain in the in-plane film dimension. Formation of the charged head-to-head domain wall, however, reduces the internal 
electric fields compared to the head-to-head $180^{\circ}$ domain boundary, as shown in Figures $4 \mathrm{~b}$ and 4d. The largest changes occurred in the out-of-plane depolarization fields around the bottom of the $P_{[00 \overline{1}]}$ domain, which both decreased in magnitude after formation of the charged domain wall and changed sign. Similar, but smaller, reductions in the magnitude of the in-plane component of the depolarization field around the lateral side of the switched domain also occurred, but after the formation of the charged domain wall an increased depolarization field was created in the preexisting $P_{[\overline{100}]}$ domain due to bound charges at the head-to-head interface. The observed reductions in the depolarization field around the $P_{[00 \overline{1}]}$ domain structure, however, may explain the stability of the domain structure during switching at moderate biases.

The electrostatic energy densities around the switching domain structure both before and after formation of the charged domain wall are plotted in Figures 4e and 4f, respectively. Removal of part of the depolarizing electric field through formation of the charged domain wall reduced the electrostatic energy density around the charged $90^{\circ}$ domain wall compared to the charged $180^{\circ}$ domain wall. Large reductions in the energy density occurred where the out of plane depolarization field was removed as described previously. In contrast, increases in the electrostatic energy density occurred within the ferroelastic domain adjacent to the switched $P_{[00 \overline{1}]}$ domain through the formation of a modest depolarization field. Investigation of the net change in the electrostatic energy density showed, however, that the total electrostatic energy around the domain structure decreased due to these changes in the depolarization fields.

Reductions in the energy are also partially due to the fact that formation of charged $90^{\circ}$ domain walls moves the charged wall plane from the (001) plane to the (101) plane[47] and therefore reduces the total bound charge at the domain boundary. To qualitatively explain this change we note that for the $180^{\circ}$ domain boundary the change in the normal polarization at the 
charged wall is $2 P_{s}$ where $P_{s}$ is the spontaneous polarization in the system. When the wall is rotated by $45^{\circ}$, however, the change in the component of the polarization normal to the domain wall is reduced to $2 P_{S} \operatorname{Cos}\left(\frac{\pi}{4}\right)$, representing a large decrease in the charge at the domain wall of $0.585 P_{s} .[12]$ Additional reductions in the electrostatic energy may also result from the considerable broadening of the charged $90^{\circ}$ domain wall in response to the bound charge that distributes the charge over a wider area, contributing to the reduced depolarization fields.

While the depolarization fields are accommodated at the charged $90^{\circ}$ domain wall, additional elastic stresses were formed at the boundary to accommodate the lattice stresses induced by the broadened domain wall. Figure 5 shows that the in-plane and out-of-plane components of the dilatational stress are increased at the charged domain wall compared to the uncharged $90^{\circ}$ domain wall. The largest increases in the in-plane $\sigma_{11}$ component of the stress (Figures $5 \mathrm{a}$ and $5 \mathrm{~b}$ ) occurred in the broadened domain wall where the magnitude of the polarization along the body diagonal was largest, indicating the broadened domain wall and gradual rotation of the polarization across the wall reduces the elastic compatibility predicted for a sharp $90^{\circ}$ domain wall on the (101) plane.[47] In contrast, the out-of-plane $\sigma_{33}$ component of the stress at the center of the charged domain boundary is reduced compared to the uncharged ferroelastic domain boundaries as shown in Figures 5c and 5d. This stress, however, remained elevated at the edges of the charged domain boundary. Along with the increased $\sigma_{11}$ stress, the elevated stress at the edges of the charged domain boundary indicate that while the domain wall broadening reduced the electrostatic energy of the system, it increased the overall local elastic energy density.

As stress around the ferroelastic domain is seen to play such a critical role in the formation of the diffuse charged $90^{\circ}$ domain wall it is worth considering the impact of changing 
the overall stress state of the thin film. This can be accomplished either by releasing the substrate through various patterning techniques $[1,48]$ or through growing the film sufficiently thick that most or all of the coherency strain in the film is released.[49] Average strain in a thin film can be approximated using the domain fraction and average stress in each domain $\bar{\varepsilon}=$ $f_{a}\left(a_{a}-a_{0}\right)+f_{c}\left(a_{c}-a_{0}\right)[50]$ where $a_{\mathrm{c}}$ and $a_{\mathrm{a}}$ are the longer and shorter lattice constants of tetragonal PZT, $a_{0}$ is the lattice constant of the substrate, and $f_{\mathrm{a}}$ and $f_{\mathrm{c}}$ are the respective fraction of the domain in the film. Through changing domain fractions in PZT films average strain in the thin film is adjusted toward zero. This two domain mixture is stable room temperature over coherency strains between roughly $0.8 \%$ and $-0.8 \%$. Within this range making the coherency strain less negative leads to an increase in the domain fraction that may [27] eliminate a nearly all of the average strains in the film. [50] Modifying the compressive strains in the film by reducing or eliminating the coherency strain changes the domain fraction in the system, however, will not change the local strain states around the charged head-to-head domain wall. These local mechanical strains are seen to develop as a consequence of the interactions between local bound charges and mechanical stresses in the ferroelectric rather than as a result of a change or removal of the coherency strain or surface constraint. Consequently, the switching pathway described here is likely to be observed around isolated ferroelastic domains for a wide range of mechanical constraints applied to the film.

While the switching path and formation of a diffuse charged interface are in good agreement with previously reported results, a different domain structure was observed in the diffusion interface. In contrast to previous results showing the charged $90^{\circ}$ interface was stabilized by formation of a broad region described as a glassy dipole structure with many randomly oriented micro-domains each with only a few unit cells in extent[12] in this simulation 
the polarization changed orientation smoothly and remained nearly constant in magnitude across the interface. The more ordered structure observed in the simulation is likely a lower energy structure than the glassy dipole structure since rapid random changes in the polarization direction are avoided. This eliminates the elevated concentration of high energy domain wall-like interfaces present in the glassy dipole structure. In fact, both simulations[43] and HRTEM observations of static charged $90^{\circ}$ interfaces in PZT thin film in Figure 3 and Refs. [38,44] also observed rotations in the polarization distribution to the body diagonal at the interface rather than formation of a disordered dipole region. This suggests that the glassy dipole region is only accessible at certain conditions far from the near equilibrium conditions in the simulation. For instance, formation of a glassy dipole region may occur when the polarization is rapidly switched, kinetically hindering the ability of the system to form a charged domain wall with a smoothly changing polarization. When the $90^{\circ}$ is free to approach the lowest energy state, however, as in phase field simulations and at quasi-static domain walls, the $90^{\circ}$ charged interface observed here is likely preferred.

We next investigated the stability of the charged domain wall to back switching since these charged domain walls are implicated in the instability of written domain structures in PZT films. When the applied electric field was removed the charged domain wall gradually switched back to the original domain configuration, as shown in Figure 6. As the domain structure relaxed, the switched domain was observed to shrink in lateral extent and preserve the charged domain wall until the switched domain was nearly removed. As a result, it is concluded that the broadened wall created during switching is intrinsically not stable without the applied electric field. This behavior is likely due to the reduced electrostatic energy density (Figure 4) and increased stresses around the charged $90^{\circ}$ domain wall. Formation of a charged $180^{\circ}$ domain 
wall created by directly removing the charged $90^{\circ}$ domain wall would require formation of strong depolarizing electric fields during back switching. Rather, back switching appears to be driven by reduction of the elastic stresses created by the broad domain wall around the switched domain. Figure 6 shows the domain retreats from the edge of the charged domain wall where the stresses $\sigma_{11}$ and $\sigma_{33}$ were found to be largest (Figure 5), indicating elastic stress reduction is most likely the primary driving force for polarization back switching at charged domain walls.

Persistent charged $90^{\circ}$ domain walls that have been previously reported [12] and observed in this study were likely stabilized by elastic stress fields around defects such as oxygen vacancies or internal dislocations,[51,52] which have been shown to inhibit domain wall motion[2,3] and could reasonably compensate for stresses around the charged domain wall. Measuring the defect concentration near the domain boundary, however, was beyond the scope of this study. Point defects such as oxygen vacancies, however, have been reported to occur with charged domain walls and screen local bound charges, further increasing the stability of the charged domain wall.[52] Without a similar stabilization mechanism present in the simulation, however, back switching readily occurred at the charged ferroelastic domain walls. This tendency for back switching without an additional stabilizer may explain previously reported instability of written domain structures in PZT thin films.[17]

Ferroelectric switching through the preexisting ferroelastic domain continued only at higher applied biases via $180^{\circ}$ switching of the ferroelastic domain in the phase field simulation, as shown in Figure 7. These results show that immobile ferroelastic domain walls strongly inhibit motion of $180^{\circ}$ ferroelectric domain walls through the system, in agreement with previous experimental results. $[9,12,13,17]$ As the applied bias was increased, however, the depolarization field at the upper edge of the charged $90^{\circ}$ domain wall increased until local 
nucleation of a switched ferroelastic $P_{[100]}$ domain occurred (Figure 7a), forming a new $180^{\circ}$ charged domain wall that rapidly progressed through the existing ferroelastic domain. Formation of the $P_{[100]}$ domain also removed the charged interface between the $P_{[00 \overline{1}]}$ and $P_{[\overline{1} 00]}$ domains and resulted in the formation of a narrow, charge neutral $90^{\circ}$ domain wall typical of a mechanically and electrically compatible $90^{\circ}$ interface. As the $P_{[100]}$ domain reached the existing $P_{[001]}$ domain, switching again abruptly stopped and a new charged $90^{\circ}$ domain wall was created (Figure 7b). Further switching occurred by coupled lateral expansion of the $P_{[100]}$ and $P_{[00 \overline{1}]}$ domains to the free surface of the thin film (Figure $7 \mathrm{c}$ ). Continued $180^{\circ}$ switching through the film required nucleation of a new $P_{[00 \overline{1}]}$ domain at a higher bias that then extended vertically through the thin film with limited lateral expansion of the domain (Figure 7d). Switching is not observed to occur by nucleation of $P_{[00 \overline{1}]}$ domains on either side of the ferroelastic domain followed by $180^{\circ}$ switching of the ferroelastic domain in the simulation as has been previously suggested.[9] This clearly shows that throughout the system ferroelastic domain boundaries inhibit $180^{\circ}$ domain wall motion and readily form partially stabilized charged interfaces.

The polarization distribution around the charged domain walls formed during $180^{\circ}$ switching of the $P_{[\overline{1} 00]}$ ferroelastic domain is shown in Figure 8. When the $P_{[100]}$ domain forms the initial diffuse domain wall between the $P_{[00 \overline{1}]}$ and $P_{[\overline{100]}}$ domains is replaced with a ferroelastic domain wall similar to the unperturbed domain walls around the preexisting ferroelastic domain. The line trace of the polarization across the domain wall shows the transition between domains occurs abruptly here over $1-2 \mathrm{~nm}$. As the $P_{[100]}$ domain extends laterally, the charged domain wall on the top side of the ferroelastic domain is completely replaced by a charge neutral domain wall. At the new charged domain wall another diffuse 
domain wall is formed, with the polarization again rotated along the body diagonal at the domain wall. As observed previously, the depolarization field at the charged domain wall was reduced by the formation of a $90^{\circ}$ domain wall with the largest depolarization field existing at the bottom edge of the charged domain wall. Nucleation of a switched $P_{[00 \overline{1}]}$ occurred at this edge of the charged domain boundary. This result indicates the formation of diffuse, charged domain boundaries that inhibit further switching at moderate applied biases is likely common in ferroelectric switching with non-uniform electric fields.

Similar switching pathways were observed during in-situ switching in the prepared PZT thin films. Figure 9 shows the continuation of ferroelectric switching after formation of the charged domain interface (Figure 3). At larger applied potentials switching occurred rapidly from the $P_{[\overline{1} 00]}$ domain to a $P_{[100]}$ domain and the $P_{[00 \overline{1}]}$ domain on the far side of the ferroelastic domain to a $P_{[001]}$ domain, converting the charged interface to three nearly charge neutral domain interfaces (Figure 3a.) This transition occurred too rapidly to directly observe in the TEM, but the simulated rapid drop in the electric field away from the electrode suggests that the electric field would not have been large enough to initiate nucleation on the far side of the domain prior to switching the $P_{[100]}$ domain.

\section{Summary}

Using a phase field model and high resolution transmission electron microscopy we have shown that charged $90^{\circ}$ domain boundaries inhibit $180^{\circ}$ domain wall motion and readily form during ferroelectric switching around preexisting ferroelastic domains in ferroelectric 
$\mathrm{Pb}\left(\mathrm{Zr}_{0.2}, \mathrm{Ti}_{0.8}\right) \mathrm{O}_{3}$ thin films. These domain walls are broader than charge neutral $90^{\circ}$ domain walls, transitioning gradually in PZT thin films between the domain states across $5-6 \mathrm{~nm}$ compared to $1-2 \mathrm{~nm}$ for uncharged $90^{\circ}$ domain walls. The distribution of the bound charge across the $90^{\circ}$ domain wall substantially reduces depolarization fields around the switched domain and this restructuring of the polarization distribution lowers the local electrostatic energy density of the switched domain structure. Continued forward switching in this domain structure was arrested and led to the formation of an additional $180^{\circ}$ charged domain wall and large depolarization fields in the thin film. We have shown that switching past the charged domain wall could be induced with a sufficiently large applied electric field, but that charged ferroelastic domain walls may form at every preexisting ferroelastic domain boundary in the thin film, resulting in numerous barriers to switching and potentially high writing biases around a ferroelastic domain. Typical ferroelectric thin films such as $\mathrm{Pb}\left(\mathrm{Zr}_{\mathrm{x}}, \mathrm{Ti}_{1-\mathrm{x}}\right) \mathrm{O}_{3}$ and $\mathrm{BiFeO}_{3}$ often contain complex ferroelastic domain structures and, as a result, may be very difficult to uniformly pole to a single domain state since the barrier to ferroelectric switching created by the formation of charged domain walls during switching may prevent uniform formation of switched domains at moderate applied biases. Rather, charged interfaces formed during writing are predicted here to lead to rapid back switching of the written domain structure as the charged domain walls transition to uncharged configurations.

\section{Acknowledgements}

This work was supported by the U.S. Department of Energy, Office of Basic Energy Sciences, Division of Materials Sciences and Engineering under Award FG02-07ER46417 (Penn State) and FG02-07ER46416 (University of Michigan). Calculations at the Pennsylvania State 
University were performed on the Cyberstar Linux Cluster funded by the National Science

Foundation through grant OCI-0821527. We also gratefully acknowledge the research groups of

C.-B. Eom at the University of Wisconsin and R. Ramesh at the University of California at

Berkeley for providing samples for TEM analysis.

\section{References}

[1] V. Nagarajan, A.L. Roytburd, A. Stanishevsky, S. Prasertchoung, T. Zhao, L.Q. Chen, et al., Dynamics of ferroelastic domains in ferroelectric thin films., Nat. Mater. 2 (2003) 43-7. doi:10.1038/nmat800.

[2] P. Gao, C.T. Nelson, J.R. Jokisaari, S. Baek, C.W. Bark, Y. Zhang, et al., Revealing the role of defects in ferroelectric switching with atomic resolution., Nat. Commun. 2 (2011) 591. doi:10.1038/ncomms 1600 .

[3] D. Su, Q. Meng, C.A.F. Vaz, M.-G. Han, Y. Segal, F.J. Walker, et al., Origin of $90^{\circ}$ domain wall pinning in $\mathrm{Pb}(\mathrm{Zr}$ 0.2, Ti 0.8)O 3 heteroepitaxial thin films, Appl. Phys. Lett. 99 (2011) 102902. doi:10.1063/1.3634028.

[4] C. Winkler, M. Jablonski, K. Ashraf, A.R. Damodaran, K. Jambunathan, J. Hart, et al., Real-Time Observation of Local Strain Effects on Nonvolatile Ferroelectric Memory Storage Mechanisms, Nano Lett. 14 (2014) 3617. http://pubs.acs.org/doi/abs/10.1021/n1501304e (accessed February 27, 2015).

[5] P. Mokrý, A.K. Tagantsev, J. Fousek, Pressure on charged domain walls and additional imprint mechanism in ferroelectrics, Phys. Rev. B. 75 (2007) 094110. doi:10.1103/PhysRevB.75.094110.

[6] W.L. Warren, D. Dimos, B.A. Tuttle, G.E. Pike, R.W. Schwartz, P.J. Clews, et al., Polarization suppression in $\mathrm{Pb}(\mathrm{Zr}, \mathrm{Ti}) \mathrm{O} 3$ thin films, J. Appl. Phys. 77 (1995) 6695. doi:10.1063/1.359083.

[7] T. Rojac, M. Kosec, B. Budic, N. Setter, D. Damjanovic, Strong ferroelectric domain-wall pinning in BiFeO3 ceramics, J. Appl. Phys. 108 (2010) 074107. doi:10.1063/1.3490249.

[8] D. Marincel, H. Zhang, A. Kumar, S. Jesse, S. V. Kalinin, W.M. Rainforth, et al., Influence of a Single Grain Boundary on Domain Wall Motion in Ferroelectrics, Adv. Funct. Mater. (2013) 1-9. doi:10.1002/adfm.201302457.

[9] J. Lee, G. Shin, K. Song, W. Choi, Y. Shin, Y.P. Park, et al., Direct observation of asymmetric domain wall motion in a ferroelectric capacitor, Acta Mater. 61 (2013) 6765-6777. doi:10.1016/j.actamat.2013.07.051.

[10] W. Li, M. Alexe, Investigation on switching kinetics in epitaxial $\mathrm{Pb}(\mathrm{Zr} 0.2 \mathrm{Ti0} .8) \mathrm{O} 3$ ferroelectric thin films: Role of the $90^{\circ}$ domain walls, Appl. Phys. Lett. 91 (2007) 262903. doi:10.1063/1.2825414.

[11] V. Nagarajan, I.G. Jenkins, S.P. Alpay, H. Li, S. Aggarwal, L. Salamanca-Riba, et al., Thickness dependence of structural and electrical properties in epitaxial lead zirconate titanate films, J. Appl. Phys. 86 (1999) 595. doi:10.1063/1.370772.

[12] P. Gao, J. Britson, J.R. Jokisaari, C.T. Nelson, S. Baek, Y. Wang, et al., Atomic-scale mechanisms of ferroelastic domain-wall-mediated ferroelectric switching., Nat. Commun. 4 (2013) 2791. doi:10.1038/ncomms3791.

[13] H. Chang, S. V. Kalinin, S. Yang, P. Yu, S. Bhattacharya, P.P. Wu, et al., Watching domains 
grow: In-situ studies of polarization switching by combined scanning probe and scanning transmission electron microscopy, J. Appl. Phys. 110 (2011) 052014. doi:10.1063/1.3623779.

[14] S. Choudhury, Y.L. Li, N. Odagawa, A. Vasudevarao, L. Tian, P. Capek, et al., The influence of $180^{\circ}$ ferroelectric domain wall width on the threshold field for wall motion, J. Appl. Phys. 104 (2008) 084107. doi:10.1063/1.3000459.

[15] L. Feigl, L.J. McGilly, C.S. Sandu, N. Setter, Compliant ferroelastic domains in epitaxial $\mathrm{Pb}(\mathrm{Zr}, \mathrm{Ti}) \mathrm{O} 3$ thin films, Appl. Phys. Lett. 104 (2014) 172904. doi:10.1063/1.4874835.

[16] T.M. Kamel, G. de With, Poling of hard ferroelectric PZT ceramics, J. Eur. Ceram. Soc. 28 (2008) 1827-1838. doi:10.1016/j.jeurceramsoc.2007.11.023.

[17] A. Roelofs, N.A. Pertsev, R. Waser, F. Schlaphof, L.M. Eng, C. Ganpule, et al., Depolarizingfield-mediated $180^{\circ}$ switching in ferroelectric thin films with $90^{\circ}$ domains, Appl. Phys. Lett. 80 (2002) 1424. doi:10.1063/1.1448653.

[18] C. Ganpule, V. Nagarajan, H. Li, a. S. Ogale, D.E. Steinhauer, S. Aggarwal, et al., Role of $90^{\circ}$ domains in lead zirconate titanate thin films, Appl. Phys. Lett. 77 (2000) 292. doi:10.1063/1.126954.

[19] M. Kohli, P. Muralt, N. Setter, Removal of $90^{\circ}$ domain pinning in (100) $\mathrm{Pb}(\mathrm{Zr} 0.15$ Ti0.85)O3 thin films by pulsed operation, Appl. Phys. Lett. 72 (1998) 3217. doi:10.1063/1.121554.

[20] C.M. Folkman, S. Baek, C.T. Nelson, H.W. Jang, T. Tybell, X.Q. Pan, et al., Study of defectdipoles in an epitaxial ferroelectric thin film, Appl. Phys. Lett. 96 (2010) 052903. doi:10.1063/1.3298362.

[21] C. Ganpule, A.L. Roytburd, V. Nagarajan, B. Hill, S. Ogale, E. Williams, et al., Polarization relaxation kinetics and $180^{\circ}$ domain wall dynamics in ferroelectric thin films, Phys. Rev. B. 65 (2001) 014101. doi:10.1103/PhysRevB.65.014101.

[22] X. Tan, J.K. Shang, Partial dislocations at domain intersections in a tetragonal ferroelectric crystal, J. Phys. Condens. Matter. 16 (2004) 1455-1466. doi:10.1088/0953-8984/16/8/026.

[23] H.-L. Hu, L.Q. Chen, Three-Dimensional Computer Simulation of Ferroelectric Domain Formation, J. Am. Ceram. Soc. 81 (1998) 492-500.

[24] L.Q. Chen, Phase-field method of phase transitions/domain structures in ferroelectric thin films: A review, J. Am. Ceram. Soc. 91 (2008) 1835-1844. doi:10.1111/j.1551-2916.2008.02413.x.

[25] Y.L. Li, S.Y. Hu, Z.K. Liu, L.Q. Chen, Effect of electrical boundary conditions on ferroelectric domain structures in thin films, Appl. Phys. Lett. 81 (2002) 427. doi:10.1063/1.1492025.

[26] S.M. Allen, J.W. Cahn, A Microscopic Theory for Anitphase Boundary Motion and its Application to Antiphase Domain Coarsening, Acta Metall. 27 (1979) 1085.

[27] Y.L. Li, S.Y. Hu, Z.K. Liu, L.Q. Chen, Effect of substrate constraint on the stability and evolution of ferroelectric domain structures in thin films, Acta Mater. 50 (2002) 395-411. doi:10.1016/S1359-6454(01)00360-3.

[28] M.J. Haun, Z.Q. Zhuang, E. Furman, S.J. Jang, L.E. Cross, Thermodynamic Theory of the Lead Zirconate-Titanate Solid Solution System, Part III: curie constant and Sixth-Order Polarization Interaction Dielectric Stiffness Coefficients, Ferroelectrics. 99 (1989) 45. http://www.tandfonline.com/doi/full/10.1080/00150198908221438 (accessed April 25, 2013).

[29] S. Choudhury, Y. Li, C. Krill III, L.Q. Chen, Phase-field simulation of polarization switching and domain evolution in ferroelectric polycrystals, Acta Mater. 53 (2005) 5313-5321. doi:10.1016/j.actamat.2005.07.040.

[30] J.X. Zhang, Y.L. Li, S. Choudhury, L.Q. Chen, Y.H. Chu, F. Zavaliche, et al., Computer 
simulation of ferroelectric domain structures in epitaxial BiFeO3 thin films, J. Appl. Phys. 103 (2008) 094111. doi:10.1063/1.2927385.

[31] M.D. Glinchuk, A.N. Morozovska, E.A. Eliseev, Ferroelectric Thin Film Properties: Peculiarities Related to Mismatch-Induced Polarization, Ferroelectrics. 314 (2005) 85-95. doi:10.1080/00150190590926201.

[32] A.N. Morozovska, S. V. Kalinin, E.A. Eliseev, V. Gopalan, S. Svechnikov, Interaction of a $180^{\circ}$ ferroelectric domain wall with a biased scanning probe microscopy tip: Effective wall geometry and thermodynamics in Ginzburg-Landau-Devonshire theory, Phys. Rev. B. 78 (2008) 1-11. doi:10.1103/PhysRevB.78.125407.

[33] A.K. Tagantsev, The role of the background dielectric susceptibility in uniaxial ferroelectrics, Ferroelectrics. 69 (1986) 321. http://www.tandfonline.com/doi/pdf/10.1080/00150198608008205 (accessed July 8, 2014).

[34] A.G. Khachaturyan, Theory of Structural Transformations in Solids, John Wiley \& Sons, New York, New York, 1983.

[35] A. Devonshire, Theory of ferroelectrics, Adv. Phys. 3 (1954) 85. http://www.tandfonline.com/doi/pdf/10.1080/00018735400101173 (accessed November 24, 2014).

[36] D.G. Schlom, L.Q. Chen, C.-B. Eom, K.M. Rabe, S.K. Streiffer, J.-M. Triscone, Strain Tuning of Ferroelectric Thin Films, Annu. Rev. Mater. Res. 37 (2007) 589-626.

doi:10.1146/annurev.matsci.37.061206.113016.

[37] L.Q. Chen, J. Shen, Applications of semi-implicit Fourier-spectral method to phase field equations, Comput. Phys. Commun. 108 (1998) 147-158. doi:10.1016/S0010-4655(97)00115-X.

[38] Y.L. Tang, Y.L. Zhu, Y.J. Wang, W.Y. Wang, Y.B. Xu, W.J. Ren, et al., Atomic-scale mapping of dipole frustration at $90^{\circ}$ charged domain walls in ferroelectric PbTiO3 films., Sci. Rep. 4 (2014) 4115. doi:10.1038/srep04115.

[39] B. Meyer, D. Vanderbilt, Ab initio study of ferroelectric domain walls in PbTiO 3, Phys. Rev. B. 65 (2002) 104111. doi:10.1103/PhysRevB.65.104111.

[40] S. Choudhury, J.X. Zhang, Y.L. Li, L.Q. Chen, Q.X. Jia, S. V. Kalinin, Effect of ferroelastic twin walls on local polarization switching: Phase-field modeling, Appl. Phys. Lett. 93 (2008) 162901. doi:10.1063/1.2993330.

[41] N. Balke, B. Winchester, W. Ren, Y.H.Y. Chu, A.N. Morozovska, E.A. Eliseev, et al., Enhanced electric conductivity at ferroelectric vortex cores in BiFeO3, Nat. Phys. 8 (2011) 81-88. doi:10.1038/nphys2132.

[42] G. Catalan, J.F. Scott, A. Schilling, J.M. Gregg, Wall thickness dependence of the scaling law for ferroic stripe domains, J. Phys. Condens. Matter. 19 (2007) 022201. doi:10.1088/09538984/19/2/022201.

[43] J. Britson, C.T. Nelson, X.Q. Pan, L.Q. Chen, First-order morphological transition of ferroelastic domains in ferroelectric thin films, Acta Mater. 75 (2014) 188-197. doi:10.1016/j.actamat.2014.04.049.

[44] P. Gao, J. Britson, C.T. Nelson, J.R. Jokisaari, C. Duan, M. Trassin, et al., Ferroelastic domain switching dynamics under electrical and mechanical excitations., Nat. Commun. 5 (2014) 3801. doi:10.1038/ncomms4801.

[45] R.J. Nelmes, W.F. Kuhs, The Crystal Structure of Tetragonal PbTiO3 at Room Temperature and at 700 K, Solid State Commun. 54 (1985) 721-723. 
[46] M.J. Hÿtch, E. Snoeck, R. Kilaas, Quantitative measurement of displacement and strain fields from HREM micrographs, Ultramicroscopy. 74 (1998) 131-146. doi:10.1016/S03043991(98)00035-7.

[47] J. Fousek, V. Janovec, The Orientation of Domain Walls in Twinned Ferroelectric Crystals, J. Appl. Phys. 40 (1969) 135. doi:10.1063/1.1657018.

[48] F. Griggio, S. Jesse, A. Kumar, O. Ovchinnikov, H. Kim, T.N. Jackson, et al., Substrate Clamping Effects on Irreversible Domain Wall Dynamics in Lead Zirconate Titanate Thin Films, Phys. Rev. Lett. 108 (2012) 157604. doi:10.1103/PhysRevLett.108.157604.

[49] Q.Y. Qiu, R. Mahjoub, S.P. Alpay, V. Nagarajan, Misfit strain-film thickness phase diagrams and related electromechanical properties of epitaxial ultra-thin lead zirconate titanate films, Acta Mater. 58 (2010) 823-835. doi:10.1016/j.actamat.2009.09.060.

[50] S.P. Alpay, A.L. Roytburd, Equilibrium Domain Structures of Epitaxial Perovskite Ferroelectric Films, MRS Proc. 474 (1997) 407-412. http://journals.cambridge.org/abstract_S1946427400313884 (accessed July 26, 2013).

[51] T. Sluka, A.K. Tagantsev, D. Damjanovic, M. Gureev, N. Setter, Enhanced electromechanical response of ferroelectrics due to charged domain walls., Nat. Commun. 3 (2012) 748. doi:10.1038/ncomms 1751 .

[52] E.A. Eliseev, A.N. Morozovska, G.S. Svechnikov, V. Gopalan, V.Y. Shur, Static conductivity of charged domain walls in uniaxial ferroelectric semiconductors, Phys. Rev. B. 83 (2011) 235313. doi:10.1103/PhysRevB.83.235313. 


\section{Figure Captions}

Figure 1 Formation of head-to-head domain wall during switching: (color online) Chronological series of images showing initial stages of switching around embedded domain as the applied potential is increased from $0 \mathrm{~V}$ to $3.0 \mathrm{~V}$. (a) The initial domain configuration contains an embedded $P_{[100]}$ within a $P_{[001]}$ domain. The marked box indicates the regions plotted in (b) - (d). (b) Nucleation and initial forward growth of a $P_{[00 \overline{1}]}$ beneath the electrode occurs at $2.5 \mathrm{~V}$. (c) As the potential is increased to 2.84 $\mathrm{V}$ the switched domain forms a charged $90^{\circ}$ domain wall that (d) broadens as the potential is further increased to $3.0 \mathrm{~V}$.

Figure 2 Structure of head-to-head domain wall: (color online) (a) The domain structure beneath the electrode tip during initial stages of $P_{[00 \overline{1}]}$ domain growth. Polarization magnitude is indicated by the color and the polarization directions projected onto the image are shown by the arrow directions. (b) Formation of the head-to-head $90^{\circ}$ domain wall results in a broad interface. Image height in (a) and (b) is $60 \mathrm{~nm}$. (c) Expanded view of charged domain wall corresponding to the region indicated by box 1 in (b). (d) Expanded view of uncharged domain wall corresponding to the region indicated by box 2 in (b). Image height in (c) and (d) is $17 \mathrm{~nm}$. Line traces along (e) the line indicated in box 1 in (b) and (f) the line indicated in box 2 in (b). Shown is the projected polarization distribution along the (101) plane (black squares), normal to the (101) plane (green circles), and the polarization magnitude.

Figure 3: In-situ observation of charged domain wall formation: (color online) Formation of a charged interface during ferroelectric switching observed with transmission electron microscopy. (a) Dark field image of the initial domain structure. (b) Dark field image of domain structure after switching with an applied electric potential of $-5 \mathrm{~V}$ above the embedded ferroelastic domain, creating a charged interface that remained stable for at least several minutes when the applied potential was removed. In (a) and (b) schematic figures beneath the dark field images show the polarization domains. (c) Map of the polarization vectors around a charged head-to-head domain wall revealing a wide transition region (purple) with gradual transition between the two polarization states (gray) accompanied by a decrease in the polarization magnitude within the transition region.

Figure 4 Electric fields and electrostatic energy density: (color online) (a),(b) In-plane electric fields around the domain structure underneath electrode tip before and after formation of the $90^{\circ}$ charged domain wall, respectively. (c),(d) Out-of-plane electric fields around the domain structures before and after the formation of the $90^{\circ}$ charged domain wall, respectively. (e),(f) Electrostatic energy density before and after formation of the $90^{\circ}$ charged domain wall, respectively.

Figure 5 Stress state around domains: (color online) (a),(b) In plane stress $\sigma_{11}$ around domain structure underneath electrode tip before and after formation of the $90^{\circ}$ charged domain wall, respectively. (c),(d) Out of plane stress $\sigma_{33}$ before and after formation of the $90^{\circ}$ charged domain wall, respectively.

Figure 6 Reverse switching of head-to-head domain wall: (color online) Chronological series of images showing domain evolution when the applied potential is removed starting from the structure in Figure 1d. (a) Initial structure. (b) - (d) Domain structure after 4 000, 7000 and 10000 time steps, respectively.

Figure $7 \mathbf{1 8 0}^{\circ}$ switching of preexisting ferroelastic domain: (color online) Chronological series of images showing domain evolution as the voltage is increased from $3.0 \mathrm{~V}$ to $7.4 \mathrm{~V}$ starting from the structure in Figure 1d. (a) As the voltage is increased to $3.7 \mathrm{~V}$ a $P_{[100]}$ ferroelastic domain is nucleated. The boxed region in (a) indicates the plotted region in (b) - (d). (b) The applied potential is increased to $3.9 \mathrm{~V}$ and the switched $P_{[100]}$ domain grows to the edge of the preexisting $P_{[100]}$ and stops. (c) As the 
applied potential is increased to $4.4 \mathrm{~V}$ the $P_{[100]}$ expands to the free surface of the thin film. (d) When the applied potential is increased to $7.4 \mathrm{~V}$ a $P_{[00 \overline{1}]}$ domain is nucleated and grows.

Figure 8 Structure around switched ferroelastic domains: (color online) (a) Domain structure around switched $P_{[100]}$ ferroelastic domain showing the formation of a narrow $90^{\circ}$ domain wall. Polarization magnitude is indicated by the color and the polarization direction projected onto the plane beneath the electrode tip is shown by the arrow directions. (b) Broad domain wall formed at intersection between $P_{[100]}$ and $P_{[001]}$ domains during switching.

Figure 9 In-Situ observation of domain switching: (color online) Continuing to apply a bias of -5 V after the formation of the charged domain wall caused in-plane switching of the embedded $\boldsymbol{P}_{[\mathbf{1 0 0}]}$ domain to $\boldsymbol{P}_{[\mathbf{1 0 0}]}$ and continued growth of the $\boldsymbol{P}_{[\mathbf{0 0 1}]}$ domain on both sides of the ferroelastic domain. Ferroelastic domain structure at $8 \mathrm{~s}$ is shown. Schematic to the right of the TEM image shows the domain configuration in the system. 
(a)

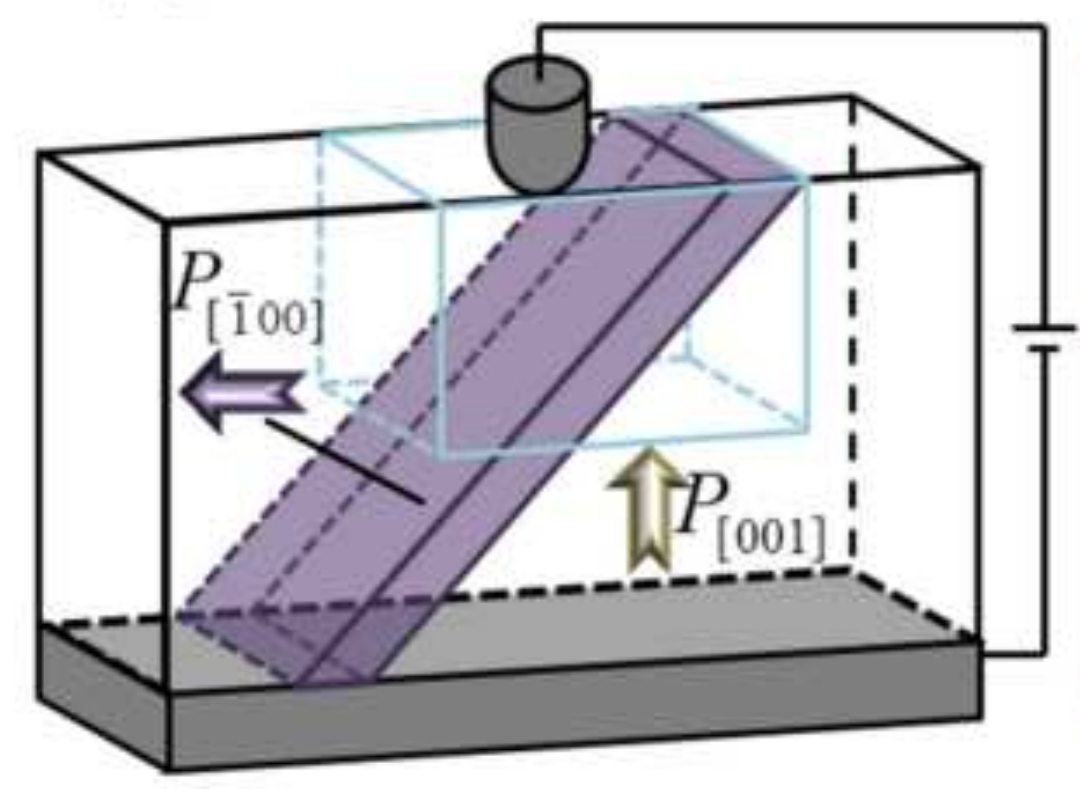

(c)

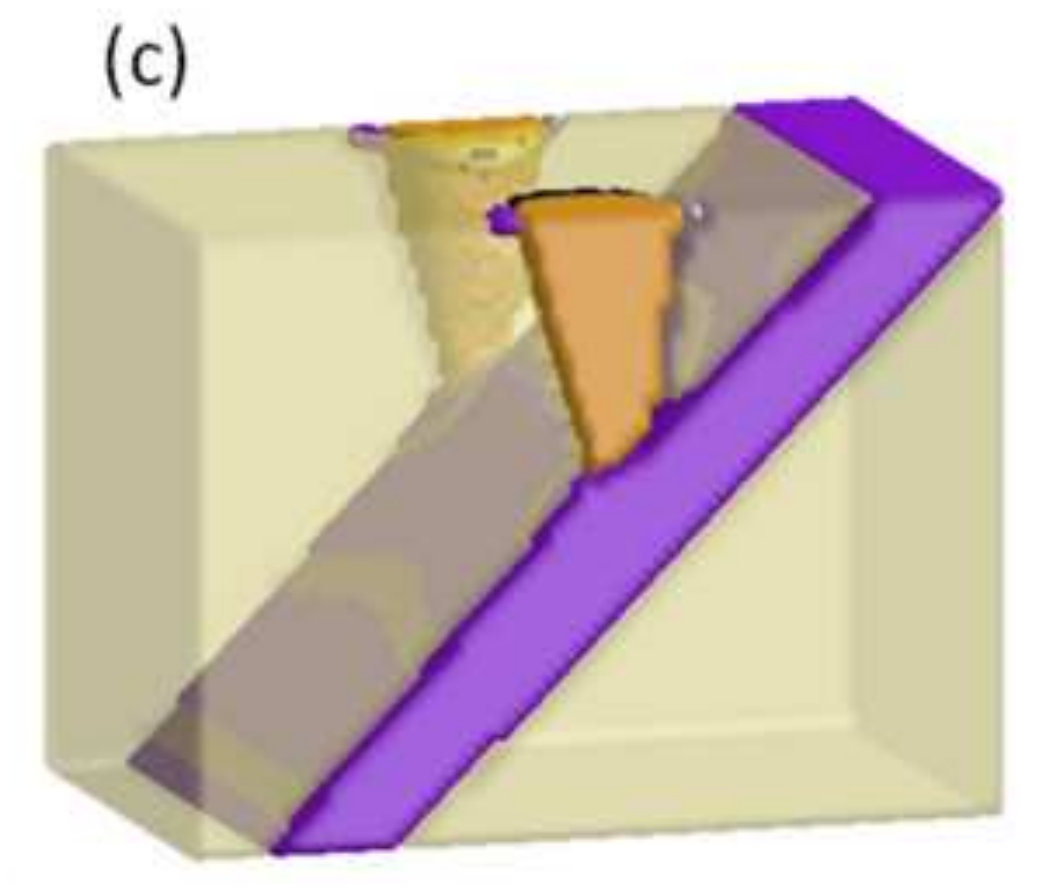

(b)

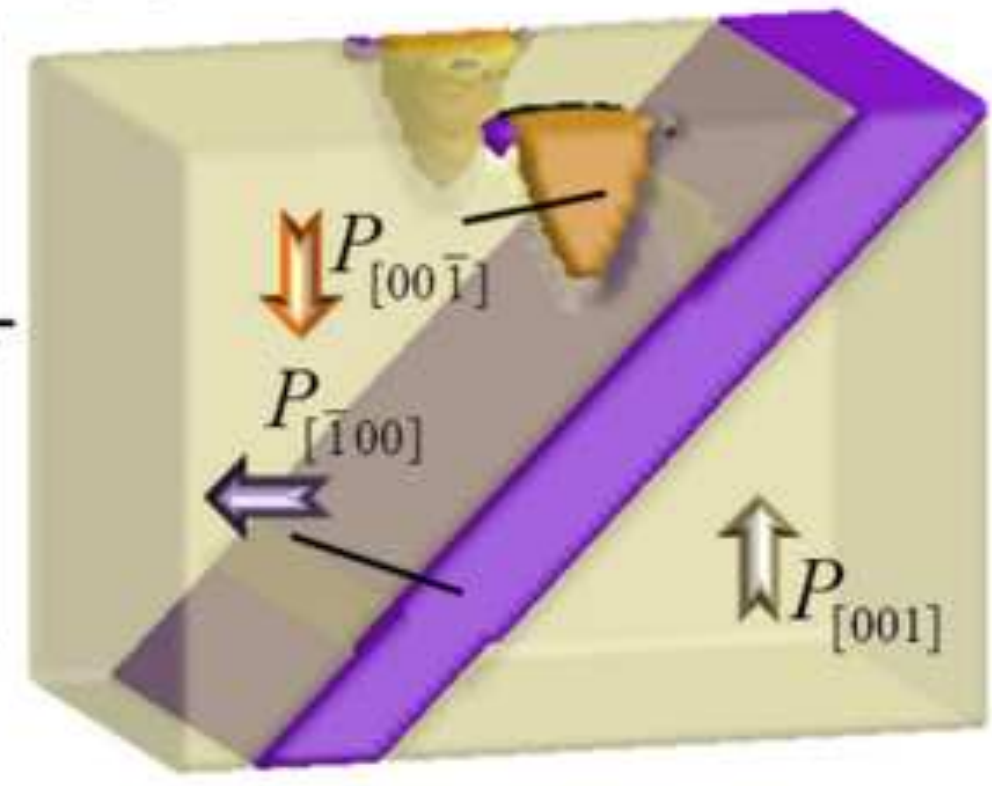

(d)

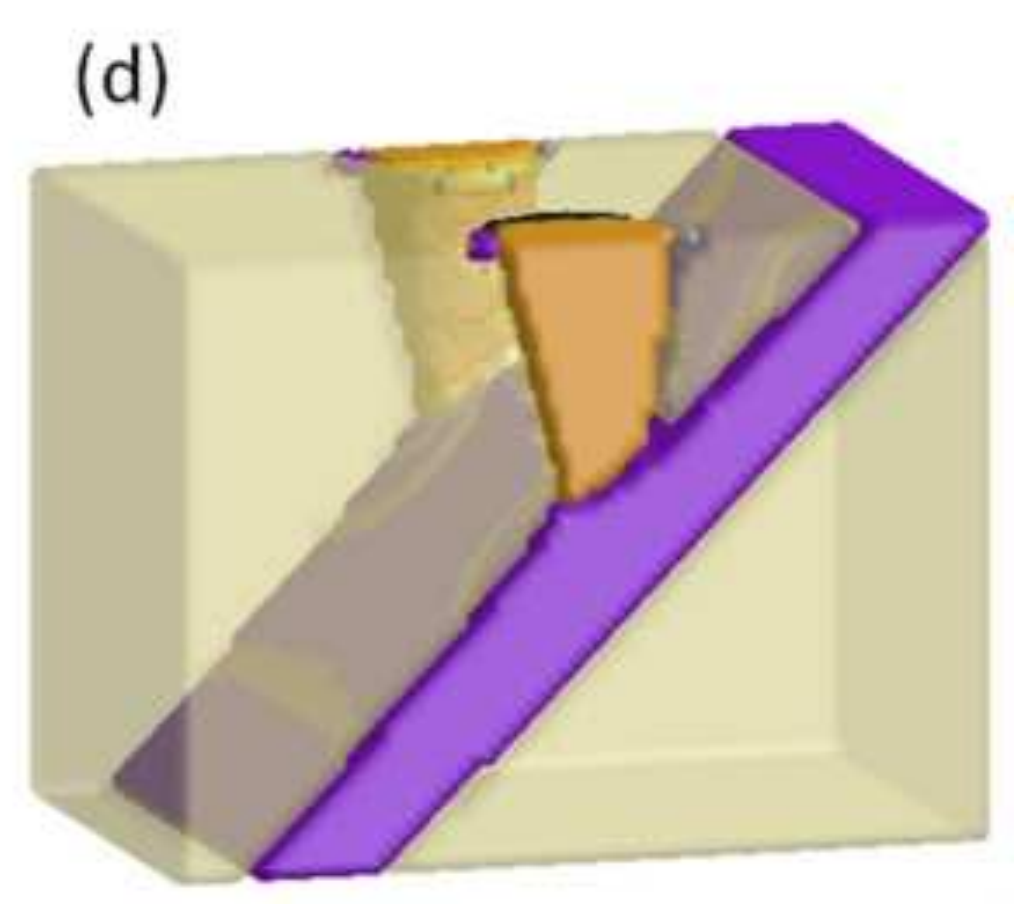


(a)

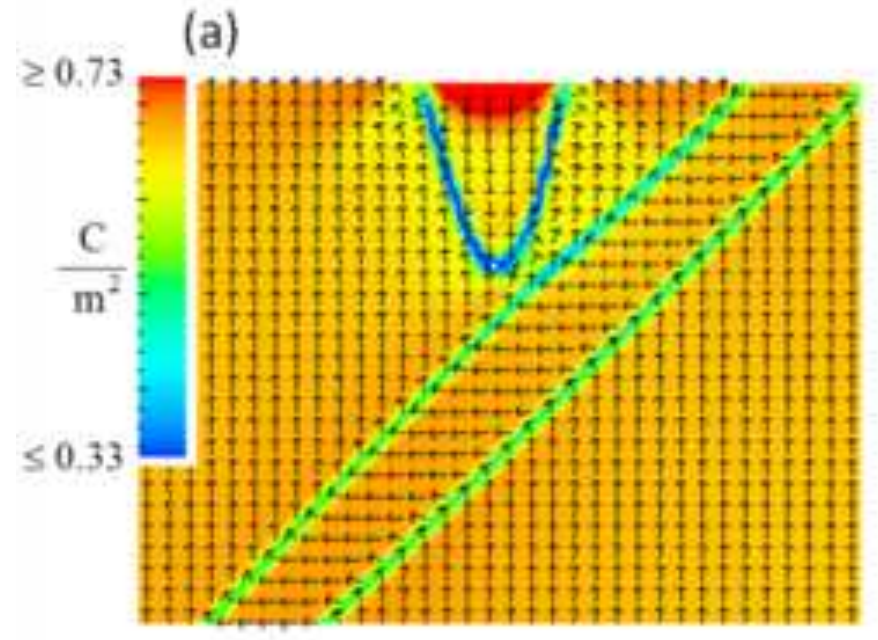

(b)

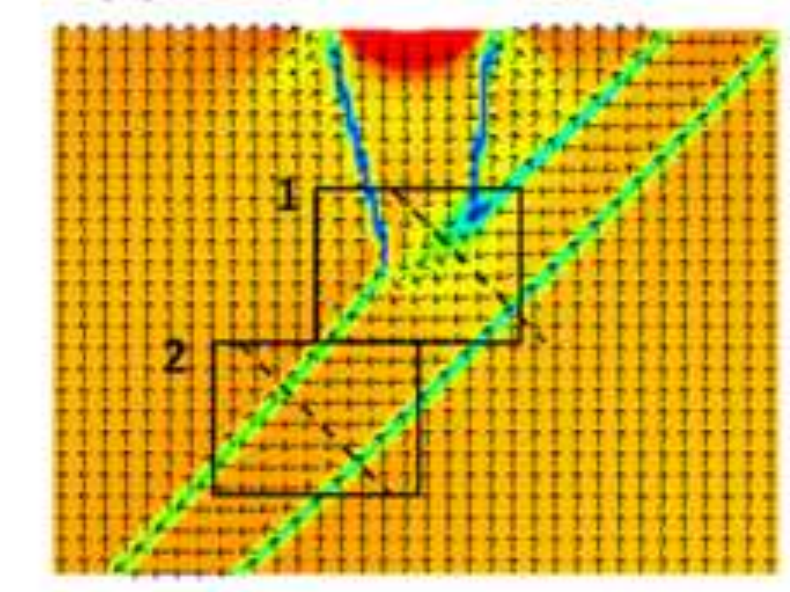

(b) (c)

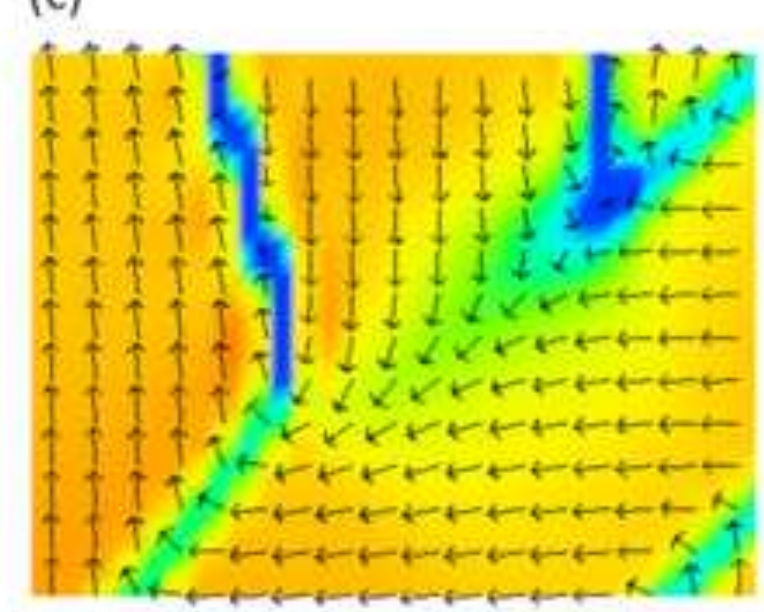

(d)

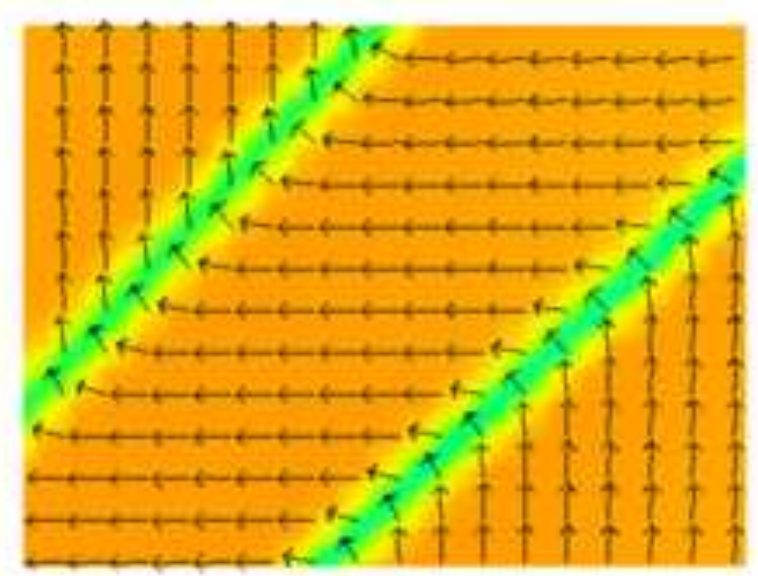

(e)
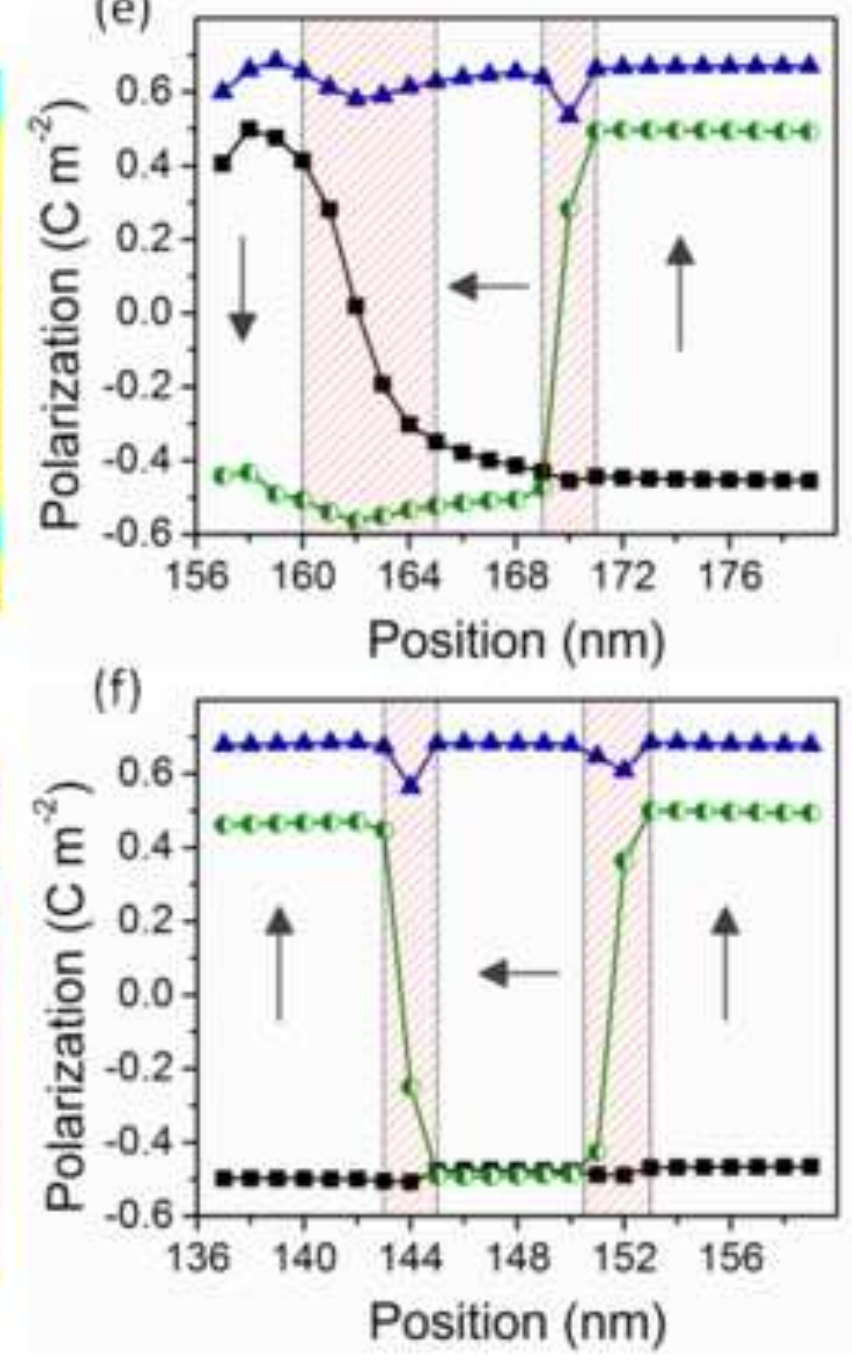
(a)
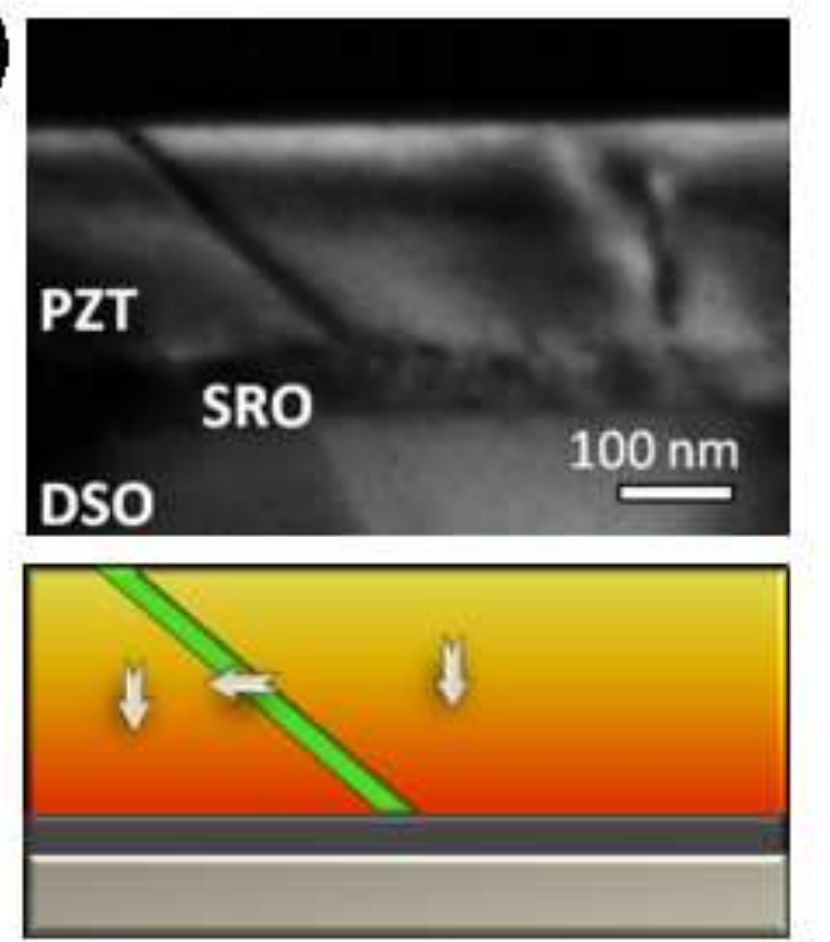

(b)
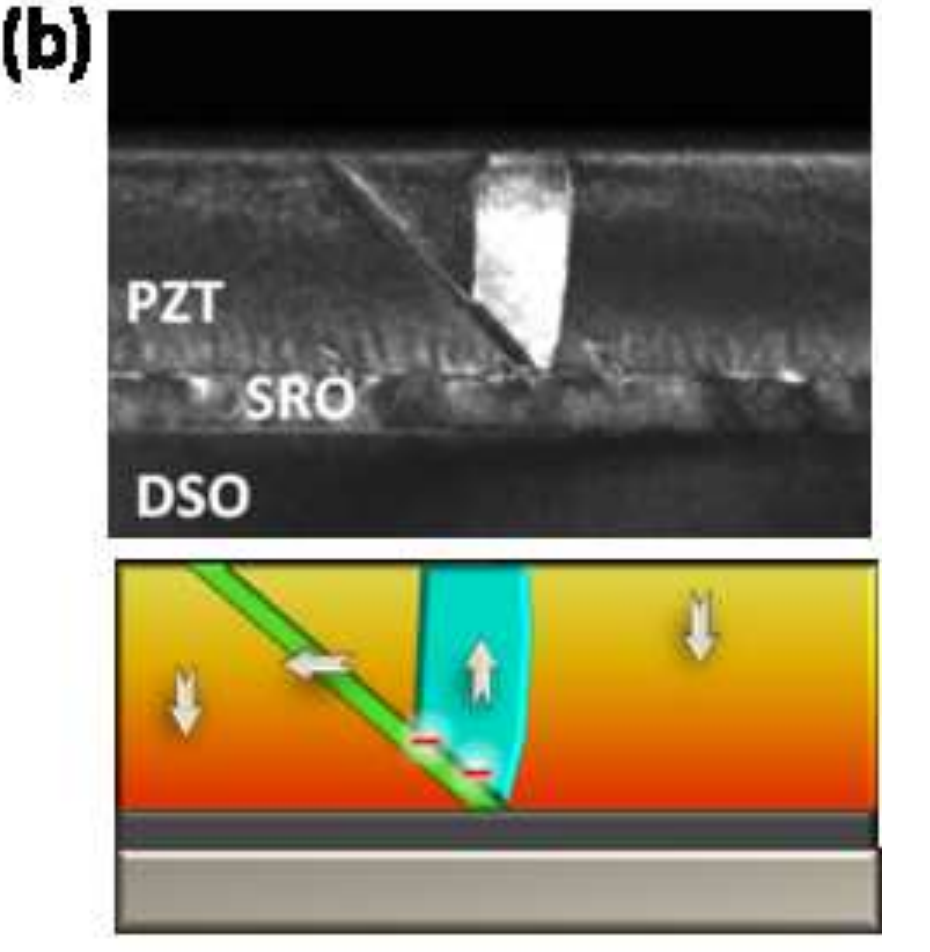

(c)
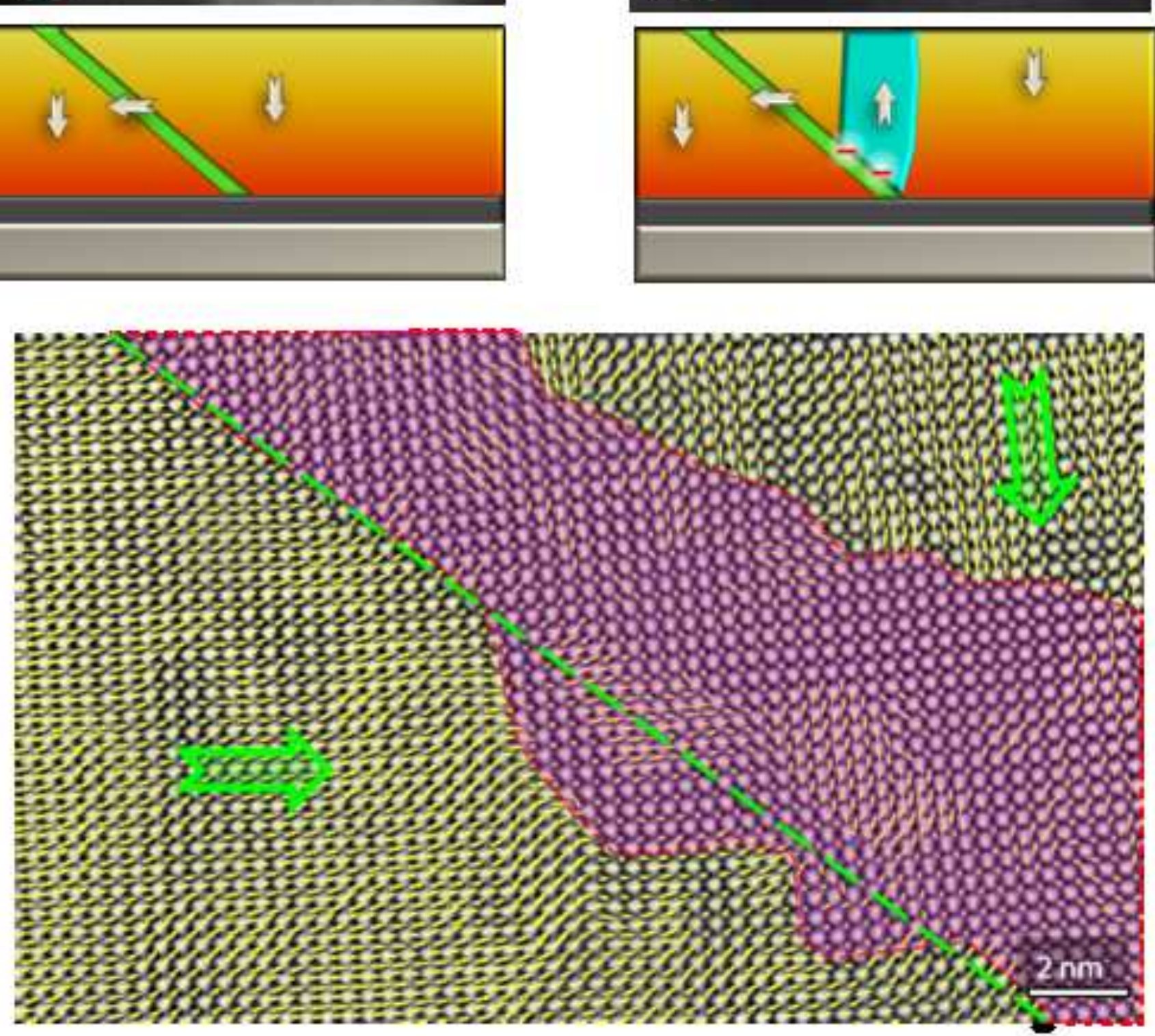

\section{.} . 

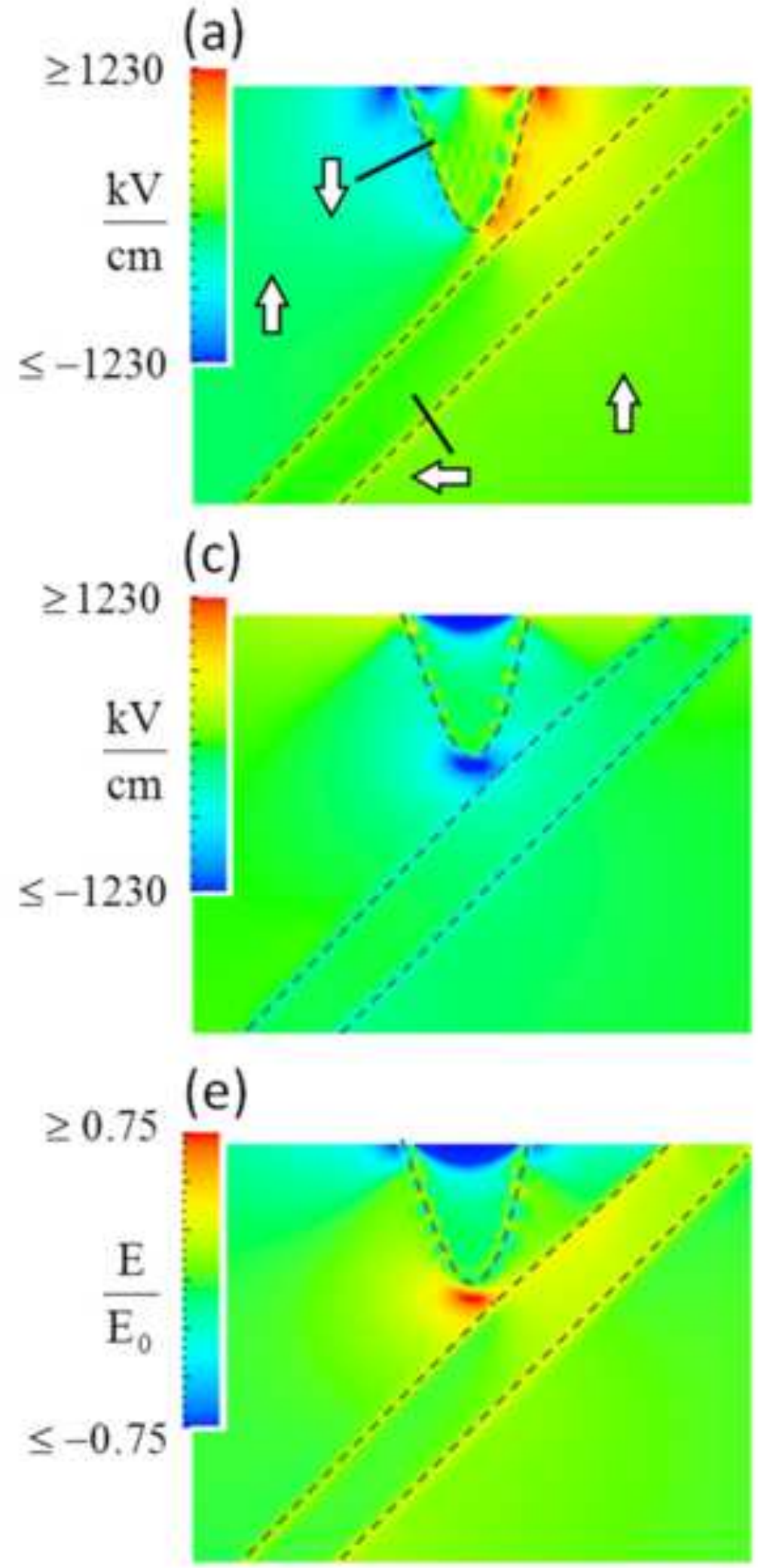

(b)

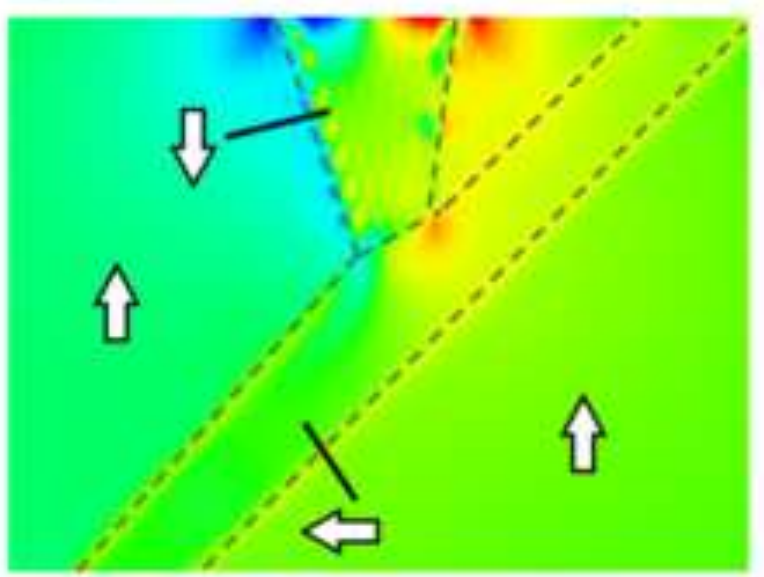

(d)

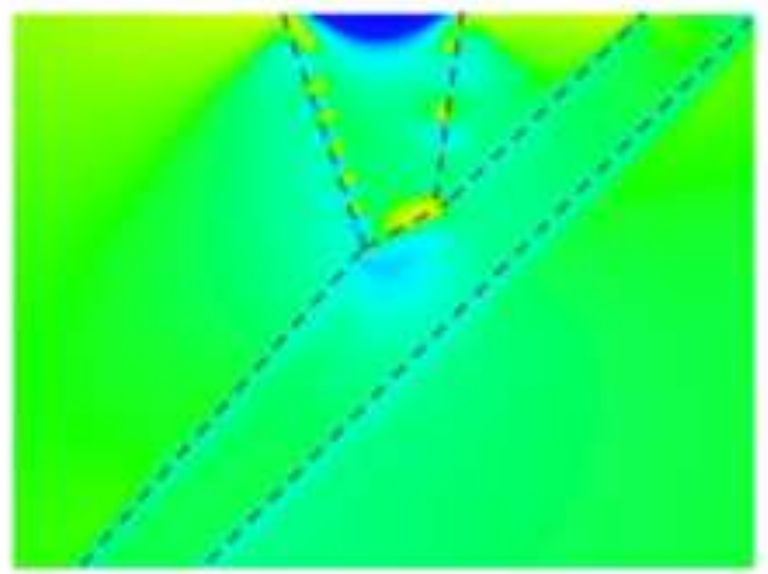

(f)

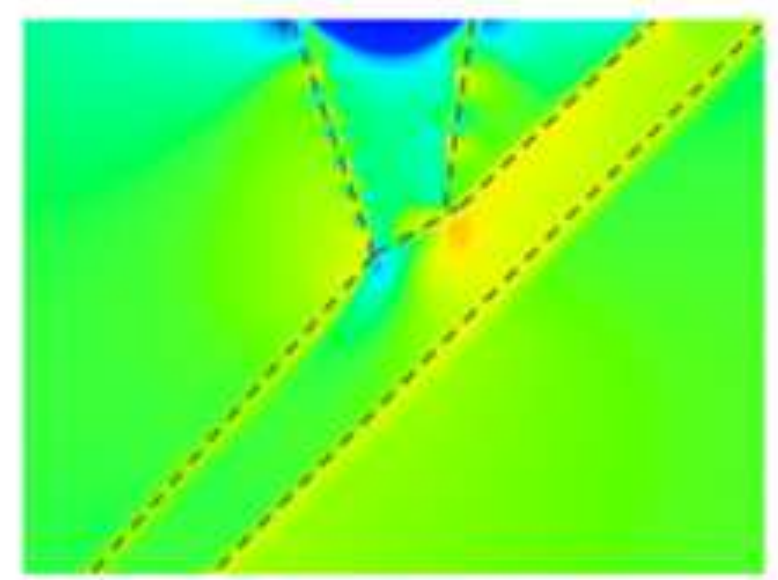




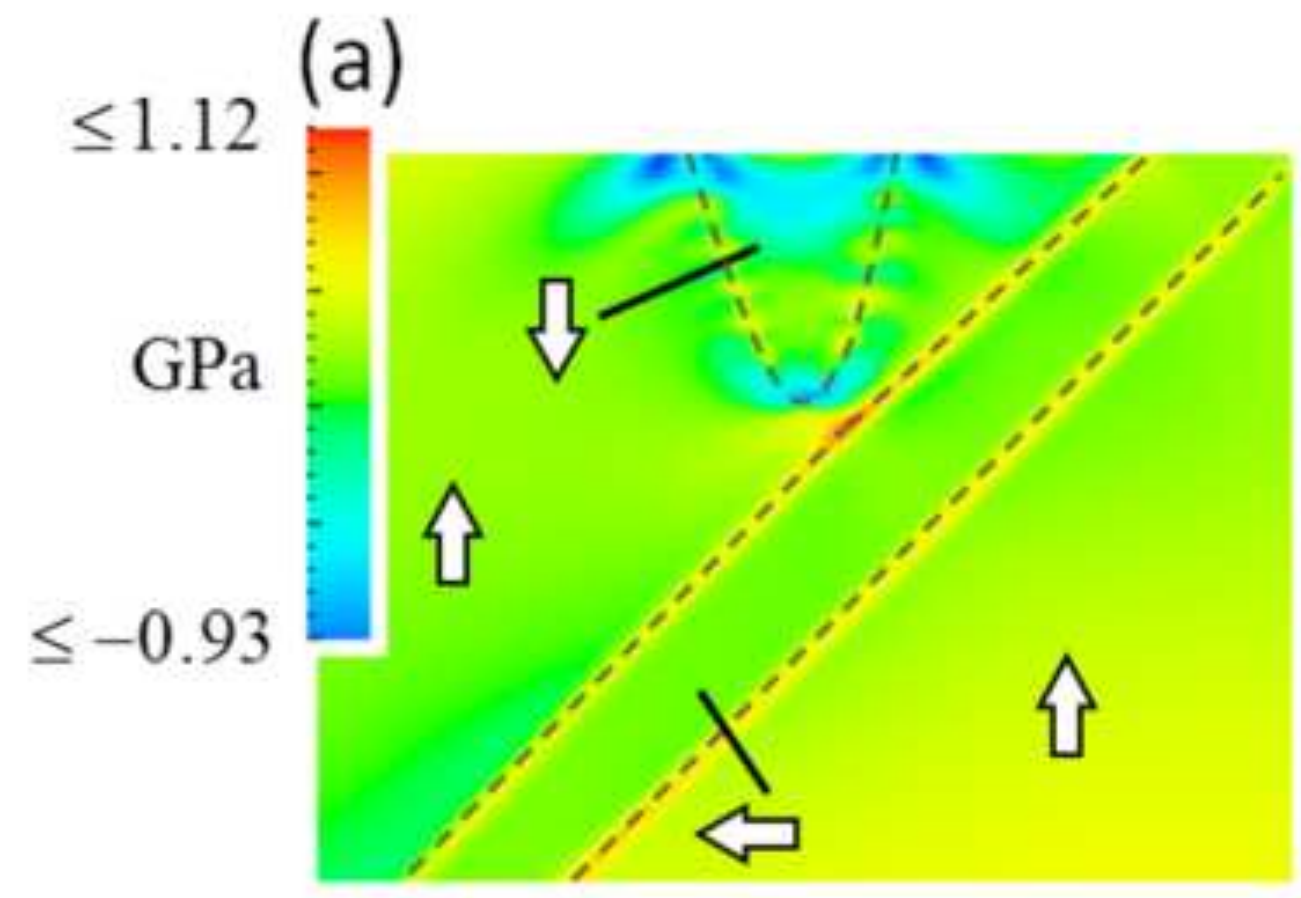

(c)

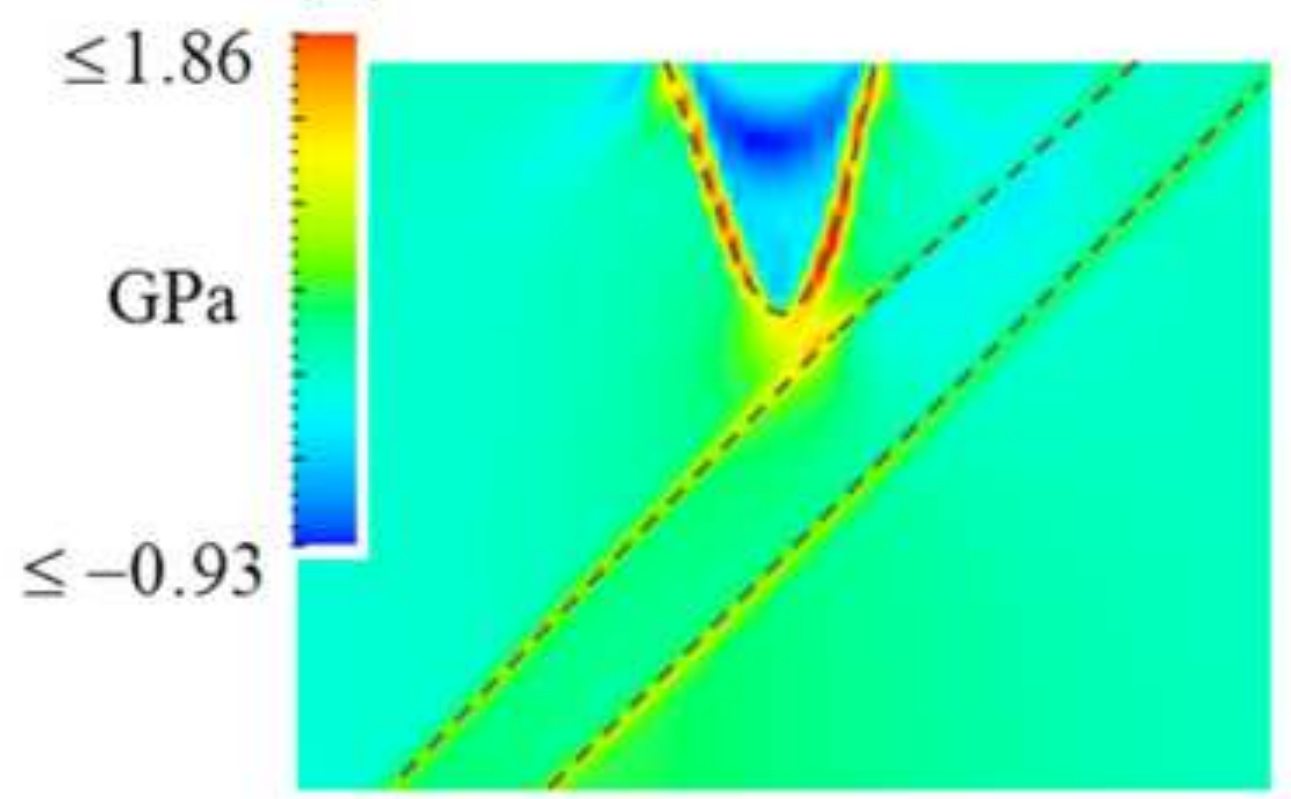

(b)

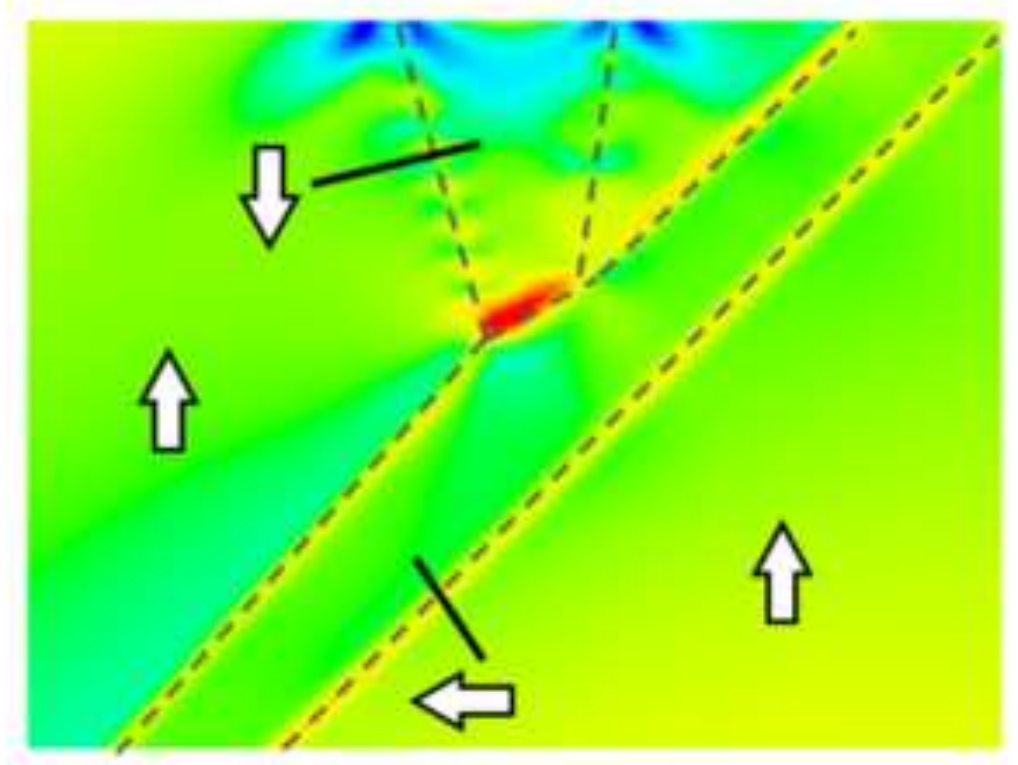

(d)

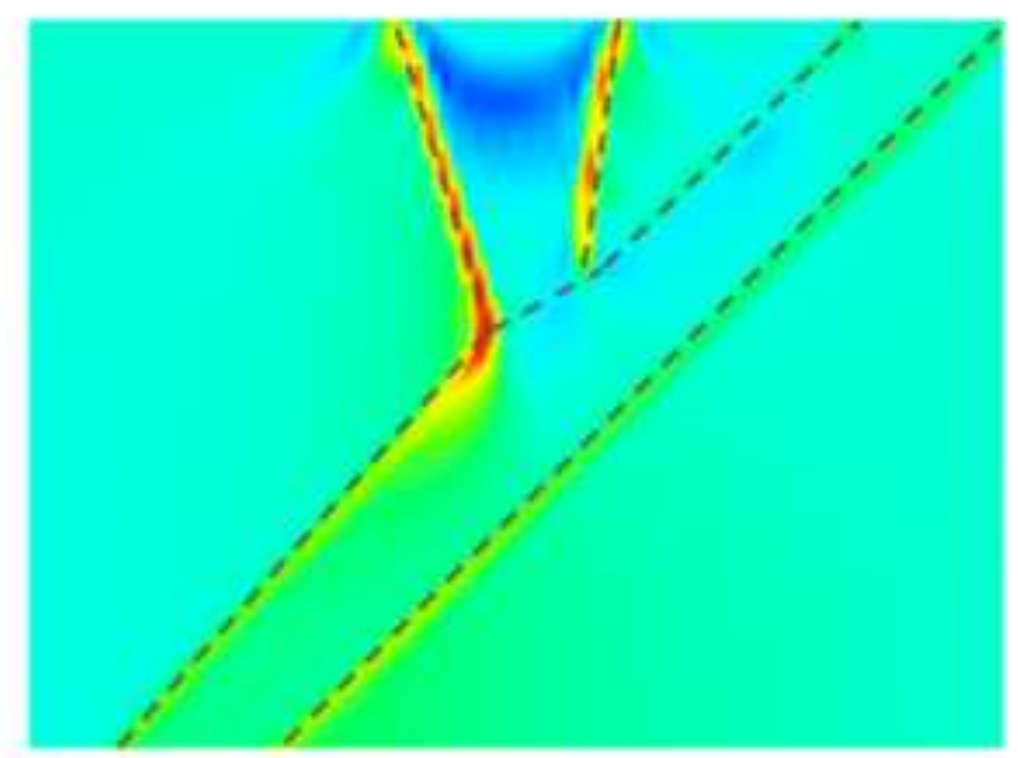


(a)

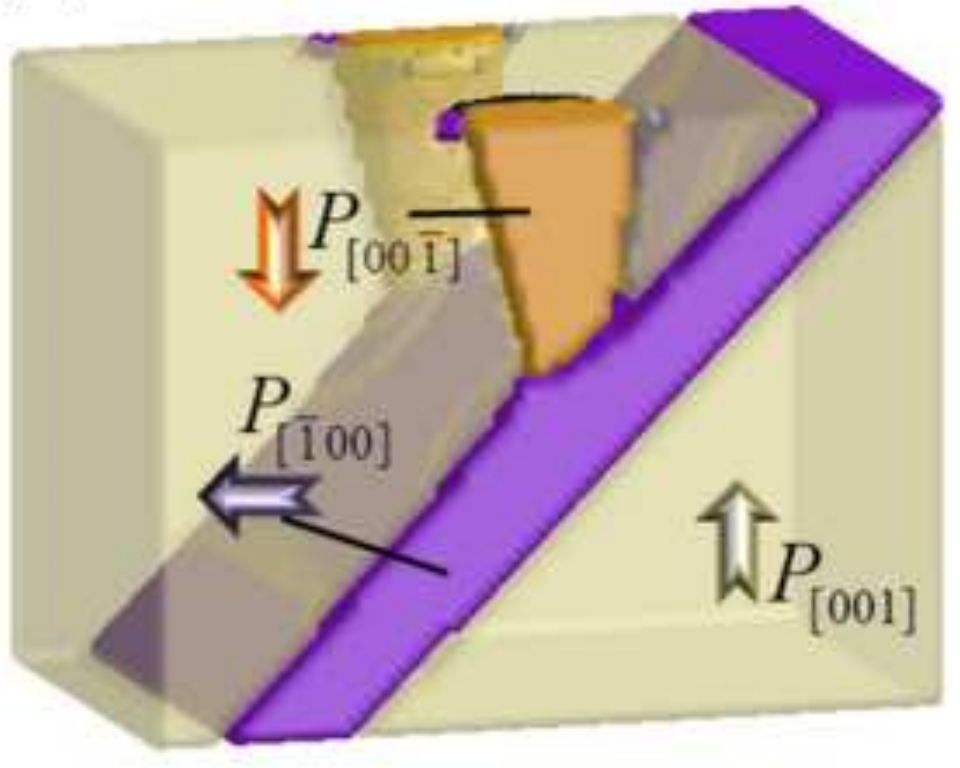

(c)

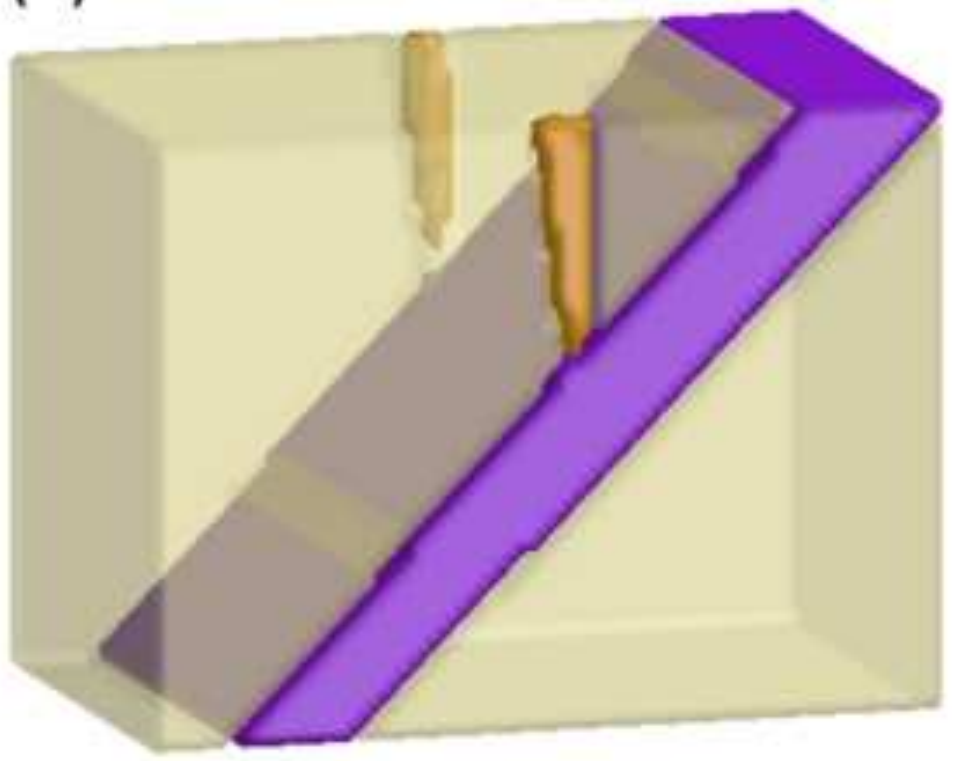

(b)

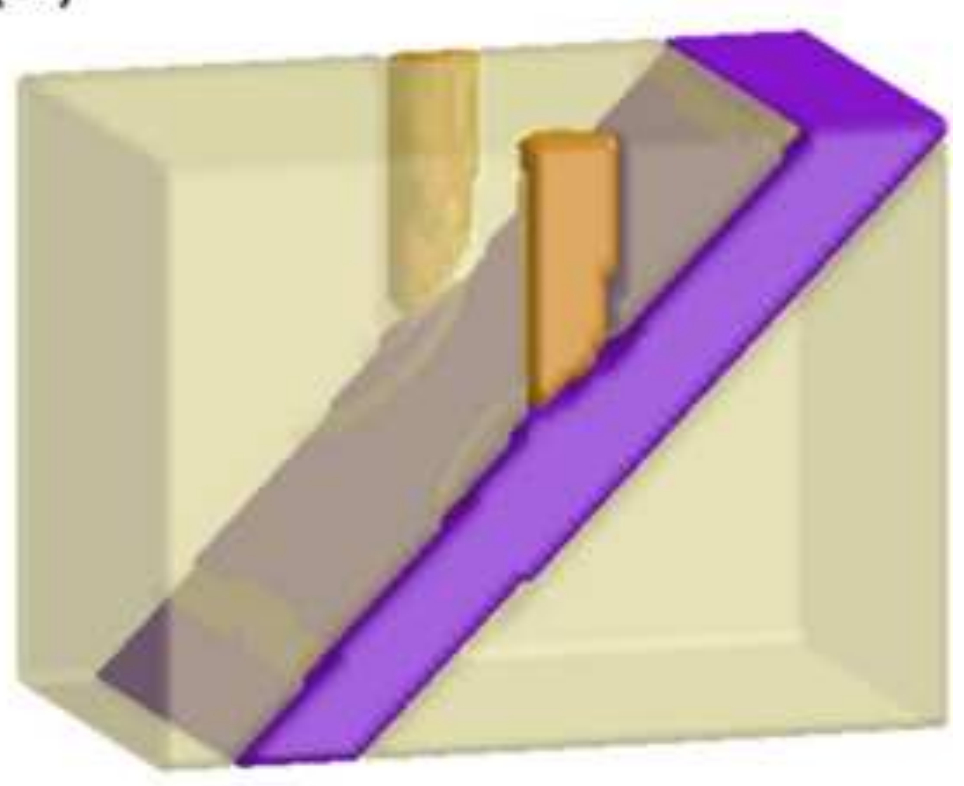

(d)

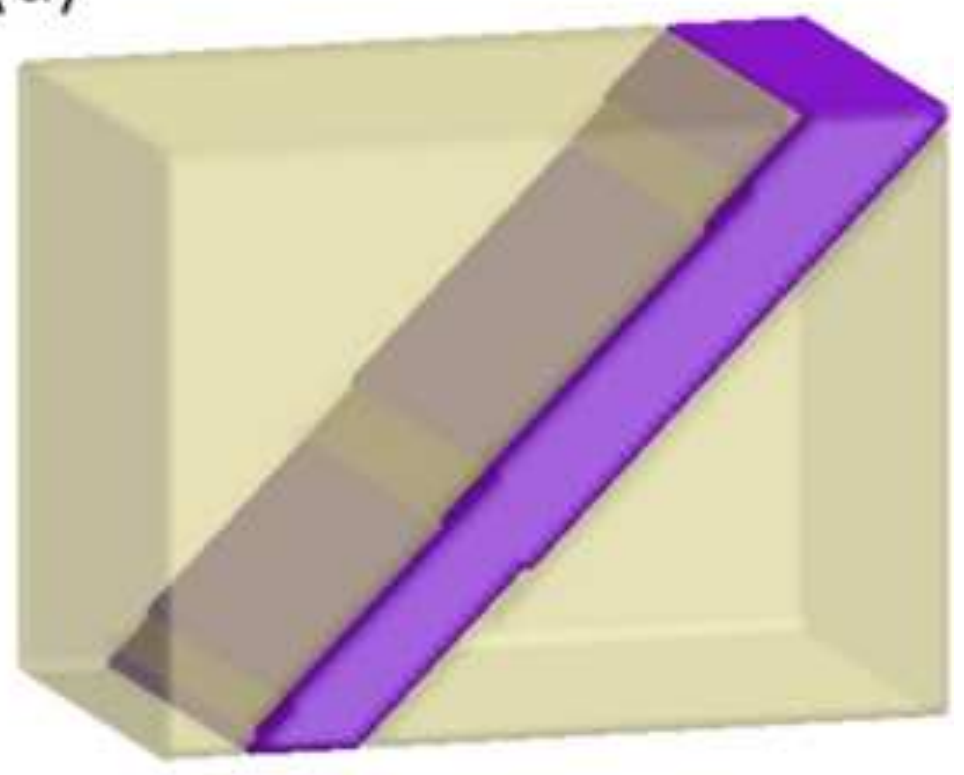


(a)

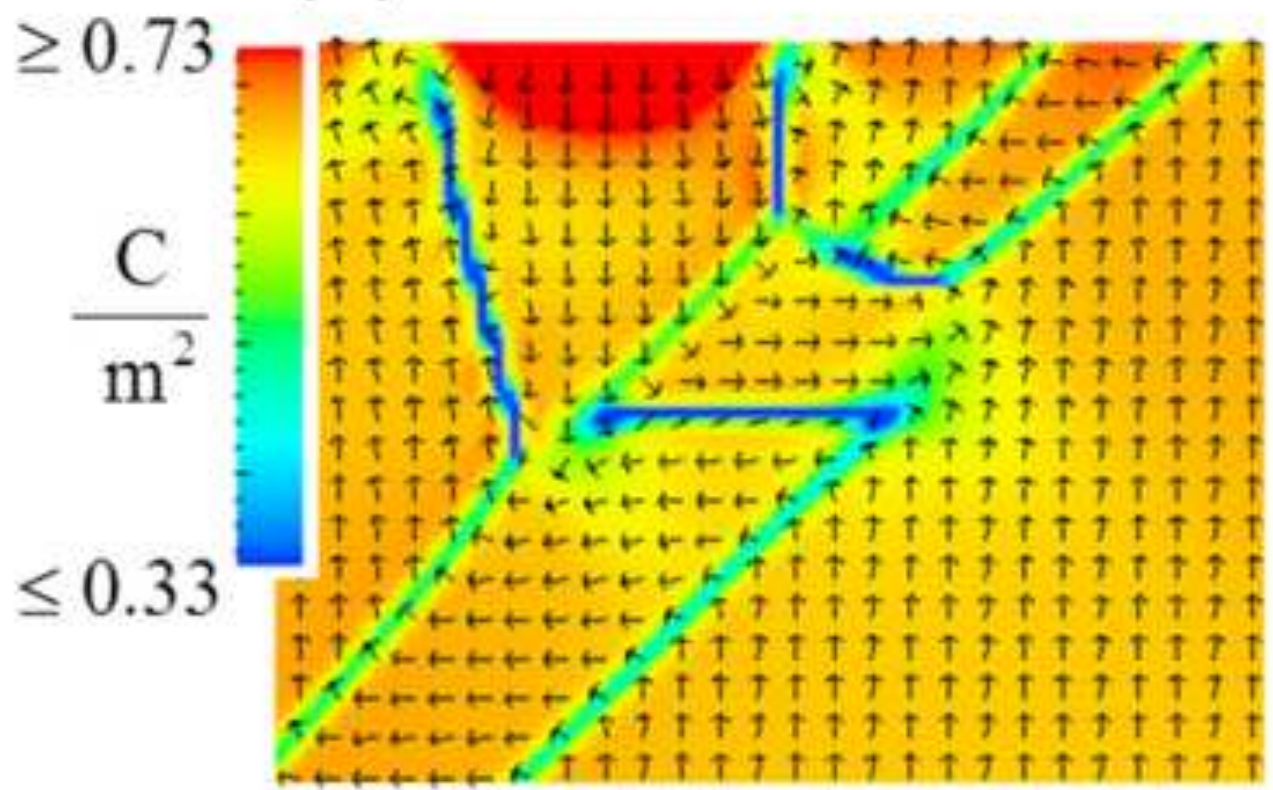

(b)

tit $x+2 \quad$ o

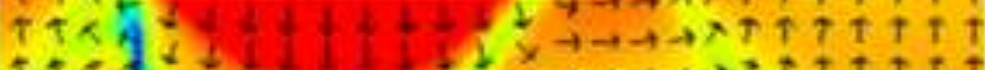

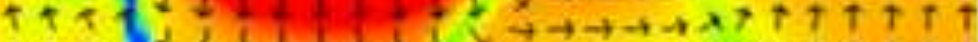

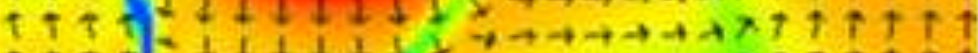

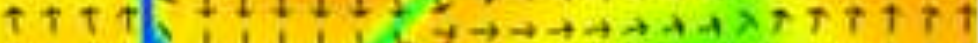

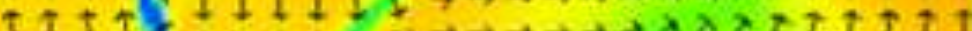

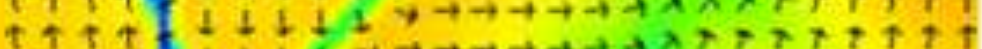

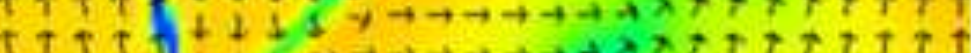

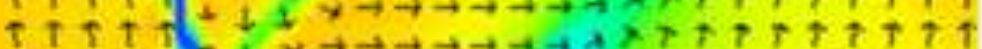

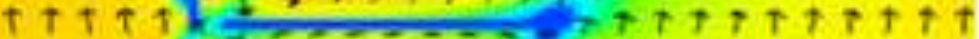

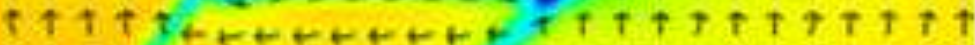
Tt T TREtEtEt

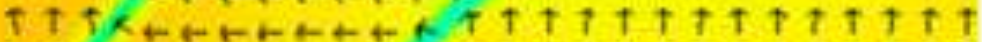

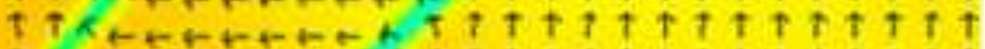

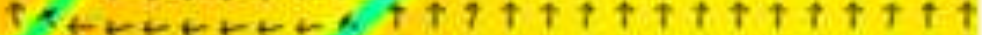

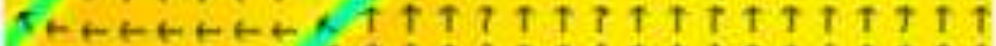

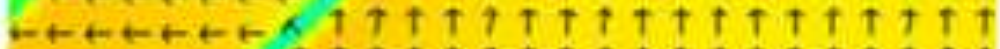

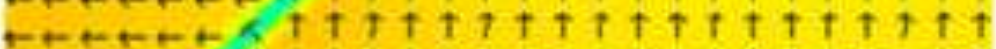
tatstititititititititit 

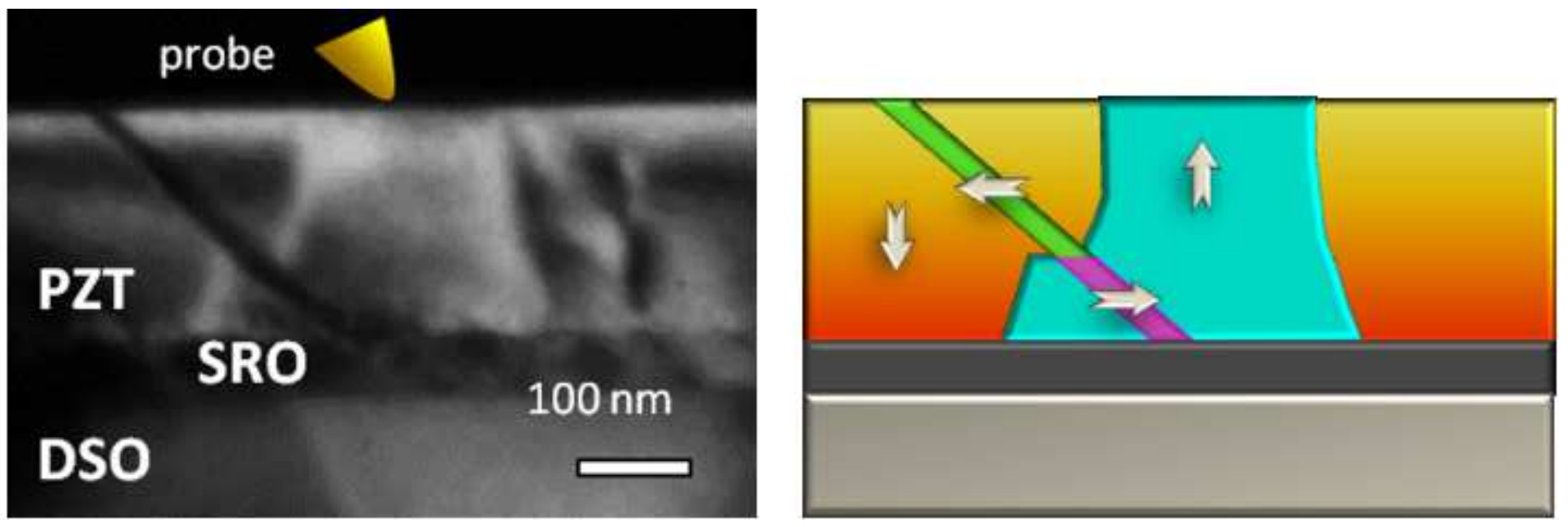


\section{Graphical Abstract Domain Polarization}
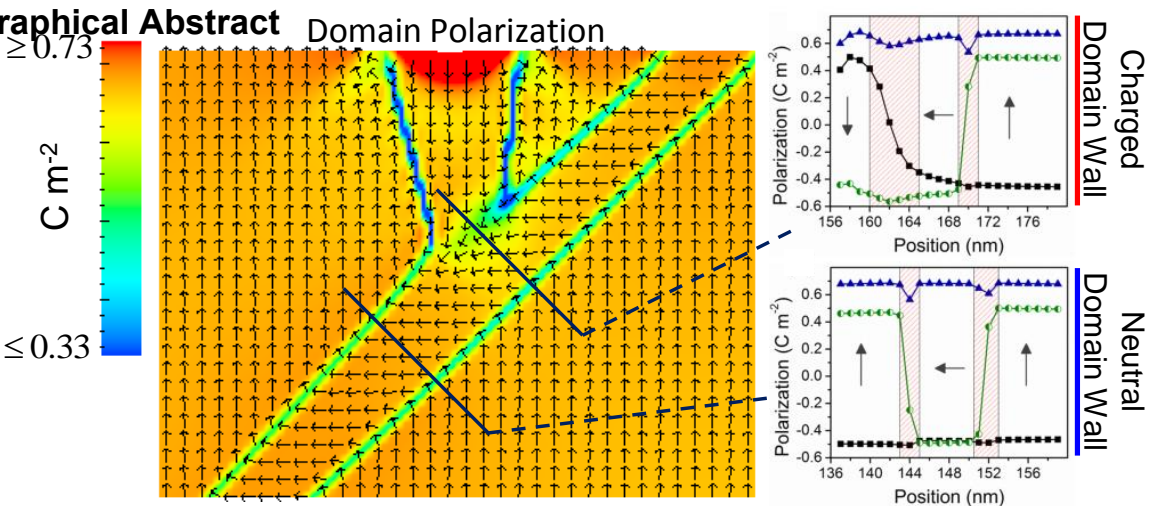

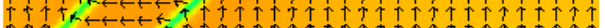

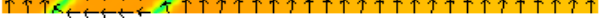
Position (nm) 